\title{
A NEW PERSPECTIVE ON ROBUST $M$-ESTIMATION: FINITE SAMPLE THEORY AND APPLICATIONS TO DEPENDENCE-ADJUSTED MULTIPLE TESTING
}

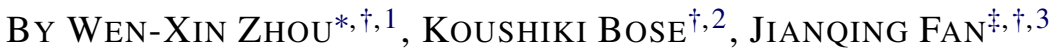 \\ AND HAN LIU ${ }^{\dagger, 4}$ \\ University of California, San Diego, * Princeton University ${ }^{\dagger}$ \\ and Fudan University
}

Heavy-tailed errors impair the accuracy of the least squares estimate, which can be spoiled by a single grossly outlying observation. As argued in the seminal work of Peter Huber in 1973 [Ann. Statist. 1 (1973) 799-821], robust alternatives to the method of least squares are sorely needed. To achieve robustness against heavy-tailed sampling distributions, we revisit the Huber estimator from a new perspective by letting the tuning parameter involved diverge with the sample size. In this paper, we develop nonasymptotic concentration results for such an adaptive Huber estimator, namely, the Huber estimator with the tuning parameter adapted to sample size, dimension and the variance of the noise. Specifically, we obtain a sub-Gaussian-type deviation inequality and a nonasymptotic Bahadur representation when noise variables only have finite second moments. The nonasymptotic results further yield two conventional normal approximation results that are of independent interest, the Berry-Esseen inequality and Cramér-type moderate deviation. As an important application to large-scale simultaneous inference, we apply these robust normal approximation results to analyze a dependence-adjusted multiple testing procedure for moderately heavy-tailed data. It is shown that the robust dependence-adjusted procedure asymptotically controls the overall false discovery proportion at the nominal level under mild moment conditions. Thorough numerical results on both simulated and real datasets are also provided to back up our theory.

1. Introduction. High dimensional data are often automatically collected with low quality. For each feature, the samples drawn from a moderate-tailed distribution may comprise one or two very large outliers in the measurements. When

Received June 2016; revised April 2017.

${ }^{1}$ Supported in part by NIH Grant R01-GM072611.

${ }^{2}$ Supported in part by NSF Grant DGE-1148900.

${ }^{3}$ Corresponding author. Supported in part by NIH Grant R01-GM072611, NSF Grant DMS1206464, and Science and Technology Commission of Shanghai Municipality under contract number $16 \mathrm{JC} 1402600$.

${ }^{4}$ Supported by NSF Grants DMS-1454377, IIS-1332109, IIS-1408910 and IIS-1546482, and NIH Grants R01-MH102339 and R01-GM083084.

MSC2010 subject classifications. Primary 62F03, 62F35; secondary 62J05, 62E17.

Key words and phrases. Approximate factor model, Bahadur representation, false discovery proportion, heavy-tailed data, Huber loss, large-scale multiple testing, $M$-estimator. 
dealing with thousands or tens of thousands of features simultaneously, the chance of including a fair amount of outliers is high. Therefore, the development of robust procedures is arguably even more important for high dimensional problems. In this paper, we develop a finite sample theory for robust $M$-estimation from a new perspective. Such a finite sample theory is motivated by contemporary statistical problems of simultaneously testing many hypotheses. In these problems, the goal is either to control the false discovery rate (FDR)/false discovery proportion (FDP), or to control the familywise error rate (FWER).

The main contributions of this paper are described and summarized in the following two subsections.

1.1. A finite sample theory of robust $M$-estimation. Consider a linear regression model $Y=\mu^{*}+\mathbf{X}^{\top} \boldsymbol{\beta}^{*}+\varepsilon$, where $\mu^{*} \in \mathbb{R}$ is the intercept, $\boldsymbol{\beta}^{*} \in \mathbb{R}^{d}$ is the vector of regression coefficients, $\mathbf{X} \in \mathbb{R}^{d}$ is the vector of covariates and $\varepsilon \in \mathbb{R}$ is the random noise variable with mean zero and finite variance. Assuming that $\varepsilon$ follows a normal distribution, statistical properties of the ordinary least squares (OLS) estimator of $\mu^{*}$ and $\boldsymbol{\beta}^{*}$ have been well studied. When the normality assumption is violated, quoting from Huber (1973), "a single grossly outlying observation may spoil the the least squares estimate" and, therefore, robust alternatives to the method of least squares, typified by the Huber estimator, are sorely needed. However, unlike the OLS estimator, all the existing theoretical results for the Huber estimator are asymptotic, including asymptotic normality [Huber (1973), Yohai and Maronna (1979), Portnoy (1985), Mammen (1989)] and the Bahadur representation [He and Shao $(1996,2000)]$. The main reason for the lack of nonasymptotic results is that the Huber estimator does not have an explicit closed-form expression, while most existing nonasymptotic analyses of the OLS estimator rely on its closed-form expression.

The first contribution of this paper is to develop a new finite sample theory for the Huber estimator. Recall the Huber loss [Huber (1964)]:

$$
\ell_{\tau}(u)= \begin{cases}\frac{1}{2} u^{2} & \text { if }|u| \leq \tau, \\ \tau|u|-\frac{1}{2} \tau^{2} & \text { if }|u|>\tau,\end{cases}
$$

a hybrid of squared loss for relatively small errors and absolute loss for large errors, where the degree of hybridization is controlled by the tuning parameter $\tau>0$ that balances robustness and efficiency. In line with this notation, we use $\ell_{\infty}$ to denote the quadratic loss $\ell_{\infty}(u)=u^{2} / 2, u \in \mathbb{R}$. Let $\left\{\left(Y_{i}, \mathbf{X}_{i}\right)\right\}_{i=1}^{n}$ be independent random samples from $(Y, \mathbf{X})$. We define the robust $M$-estimator of $\boldsymbol{\theta}^{*}:=\left(\mu^{*}, \boldsymbol{\beta}^{* \top}\right)^{\top}$ by

$$
\widehat{\boldsymbol{\theta}}:=\left(\widehat{\mu}, \widehat{\boldsymbol{\beta}}^{\top}\right)^{\top} \in \underset{\mu \in \mathbb{R}, \boldsymbol{\beta} \in \mathbb{R}^{d}}{\operatorname{argmin}} \sum_{i=1}^{n} \ell_{\tau}\left(Y_{i}-\mu-\mathbf{X}_{i}^{\top} \boldsymbol{\beta}\right) .
$$

The dependence of $\widehat{\boldsymbol{\theta}}$ or $(\widehat{\mu}, \widehat{\boldsymbol{\beta}})$ on $\tau$ will be assumed without displaying. It is worth noticing that our robustness concern is rather different from the conven- 
tional sense. In Huber's robust location estimation [Huber (1964)], it is assumed that the error distribution lies in the neighborhood of a normal distribution, which gives the possibility to replace the mean by some location parameter. Unless the shape of the distribution is constrained (e.g., symmetry), in general this location parameter is different from the mean. Our interest, however, is focused on mean estimation in the heavy-tailed case where the error distribution is allowed to be asymmetric and to exhibit heavy tails. Therefore, unlike the classical Huber estimator [Huber (1973)] which requires $\tau$ to be fixed, we allow $\tau$ to diverge with the sample size $n$ such that $\ell_{\tau}(\cdot)$ can be viewed as a robust approximate quadratic loss function. As in Fan, Li and Wang (2017), this is needed to reduce the bias for estimating the (conditional) mean function when the (conditional) distribution of $\varepsilon$ is asymmetric and heavy-tailed. In particular, by taking $\tau=\infty, \widehat{\boldsymbol{\theta}}$ coincides with the OLS estimator of $\boldsymbol{\theta}^{*}$ and by shrinking $\tau$ toward 0 , the resulting estimator approaches the least absolute deviation (LAD) estimator.

For every $\tau>0$, by definition $\widehat{\boldsymbol{\theta}}$ is an $M$-estimator of

$$
\boldsymbol{\theta}_{\tau}^{*}:=\left(\mu_{\tau}, \boldsymbol{\beta}_{\tau}^{\top}\right)^{\top}=\underset{\mu \in \mathbb{R}, \boldsymbol{\beta} \in \mathbb{R}^{d}}{\operatorname{argmin}} \mathbb{E}\left\{\ell_{\tau}\left(Y-\mu-\mathbf{X}^{\top} \boldsymbol{\beta}\right)\right\},
$$

which typically differs from the target parameter

$$
\boldsymbol{\theta}^{*}=\underset{\mu \in \mathbb{R}, \boldsymbol{\beta} \in \mathbb{R}^{d}}{\operatorname{argmin}} \mathbb{E}\left\{\ell_{\infty}\left(Y-\mu-\mathbf{X}^{\top} \boldsymbol{\beta}\right)\right\} .
$$

Note that the total error $\left\|\widehat{\boldsymbol{\theta}}-\boldsymbol{\theta}^{*}\right\|$ can be divided into two parts:

$$
\underbrace{\left\|\widehat{\boldsymbol{\theta}}-\boldsymbol{\theta}^{*}\right\|}_{\text {Total error }} \leq \underbrace{\left\|\widehat{\boldsymbol{\theta}}-\boldsymbol{\theta}_{\tau}^{*}\right\|}_{\text {Estimation error }}+\underbrace{\left\|\boldsymbol{\theta}_{\tau}^{*}-\boldsymbol{\theta}^{*}\right\|}_{\text {Approximation error }},
$$

where $\|\cdot\|$ denotes the Euclidean norm. We define $\operatorname{Bias}(\tau):=\left\|\boldsymbol{\theta}_{\tau}^{*}-\boldsymbol{\theta}^{*}\right\|$ to be the approximation error brought by the Huber loss. Proposition A.1 in the supplemental material [Zhou et al. (2018)] shows that $\operatorname{Bias}(\tau)$ scales at the rate $\tau^{-1}$, which decays as $\tau$ grows. A large $\tau$ reduces the approximation bias but jeopardizes the degree of robustness. Hence, the tuning parameter $\tau$ controls this bias and robustness trade-off of the estimator. Our main theorem (Theorem 2.1) reveals the concentration property of $\widehat{\boldsymbol{\theta}}$ and provides a nonasymptotic Bahadur representation for the difference $\widehat{\boldsymbol{\theta}}-\boldsymbol{\theta}^{*}$. Such a Bahadur representation gives a finite sample approximation of $\widehat{\boldsymbol{\theta}}$ by a sum of independent variables with a higher-order remainder. More specifically, let $\boldsymbol{\Sigma}=\mathbb{E}\left(\mathbf{X X}^{\top}\right) \in \mathbb{R}^{d \times d}$ and $\ell_{\tau}^{\prime}(\cdot)$ be the derivative function of $\ell_{\tau}(\cdot)$. Then, with properly chosen $\tau=\tau_{n}$ we have

$$
\left\|\left[\begin{array}{c}
\widehat{\mu}-\mu^{*} \\
\boldsymbol{\Sigma}^{1 / 2}\left(\widehat{\boldsymbol{\beta}}-\boldsymbol{\beta}^{*}\right)
\end{array}\right]-\frac{1}{n} \sum_{i=1}^{n} \ell_{\tau}^{\prime}\left(\varepsilon_{i}\right)\left[\begin{array}{c}
1 \\
\boldsymbol{\Sigma}^{-1 / 2} \mathbf{X}_{i}
\end{array}\right]\right\| \leq R_{n}(\tau) \simeq \frac{d}{n},
$$

where $R_{n}(\tau)$ is a finite sample error bound, which characterizes the accuracy of such a linear approximation, and $d$ is the number of covariates that may grow 
with $n$. We refer to Theorem 2.1 for a rigorous description of the result (1.4), where we obtain an exponential-type deviation inequality for this Bahadur representation.

Many asymptotic Bahadur-type representations for robust $M$-estimators have been obtained in the literature; see, for example, Portnoy (1985), Mammen (1989) and He and Shao (1996, 2000), among others. Our result (1.4), however, is nonasymptotic and provides an explicit tail bound for the remainder term $R_{n}(\tau)$. To obtain such a result, we first derive a sub-Gaussian-type deviation bound for $\widehat{\boldsymbol{\theta}}$, and then conduct a careful analysis on the higher-order remainder term using this bound and techniques from empirical process theory. The expansion (1.4) further yields two classical normal approximation results, the Berry-Esseen inequality and Cramér-type moderate deviation. These results have important applications to large-scale inference [Fan, Hall and Yao (2007), Delaigle, Hall and Jin (2011), Liu and Shao (2014), Chang, Shao and Zhou (2016)]. Consider the statistical problems of simultaneously testing many hypotheses with FDR/FDP control or globally inferring a high dimensional parameter. For multiple testing, the obtained BerryEsseen bound and Cramér-type moderate deviation result can be used to investigate the robustness and accuracy of the $P$-values and critical values. For globally testing a high dimensional parameter, the expansion (1.4), combined with the parametric bootstrap, can be used to construct a valid test. In this paper, we only focus on the large-scale multiple testing problem and leave the global testing in high dimensions for future research.

1.2. FDP control for robust dependent tests. We apply the Bahadur representation (1.4) to construct robust dependence-adjusted test statistics for simultaneous inference. Conventional tasks of large-scale multiple testing, including controlling the FDR/FDP or FWER, have been extensively explored and are now well understood when the test statistics are independent [Benjamini and Hochberg (1995), Storey (2002), Genovese and Wasserman (2004), Lehmann and Romano (2005)]. It is becoming increasingly important to understand and incorporate the dependence information among multiple test statistics. Under the positive regression dependence condition, the FDR control can be conducted in the same manner as that for the independent case [Benjamini and Yekutieli (2001)], which provides a conservative upper bound. For more general dependence, directly applying standard FDR control procedures developed for independent $P$-values may lead to inaccurate false discovery rate control and include too many spurious discoveries [Efron (2004, 2007), Sun and Cai (2009), Clarke and Hall (2009), Schwartzman and Lin (2011), Fan, Han and Gu (2012)]. In this more challenging situation, various multi-factor models have been used to investigate the dependence structure in high dimensional data; see, for example, Leek and Storey (2008), Friguet, Kloareg and Causeur (2009), Desai and Storey (2012) and Fan, Han and Gu (2012).

The multi-factor model relies on the identification of a linear space of random vectors capturing the dependence structure of the data. Friguet, Kloareg and Causeur (2009) and Desai and Storey (2012) assume that the data are drawn from a 
strict factor model with independent idiosyncratic errors. They use the expectationmaximization algorithm to estimate the factor loadings and realized factors in the model, and then obtain an estimator for the FDP by subtracting out realized common factors. These methods, however, depend on stringent model assumptions, including the independence of idiosyncratic errors and joint normality of the factor and noise. In contrast, Fan, Han and Gu (2012) and Fan and Han (2017) use a more general approximate factor model that allows dependent noise.

Let $\mathbf{X}=\left(X_{1}, \ldots, X_{p}\right)^{\top}$ be a $p$-dimensional random vector with mean $\boldsymbol{\mu}=$ $\left(\mu_{1}, \ldots, \mu_{p}\right)^{\top}$ and covariance matrix $\Sigma=\left(\sigma_{j k}\right)_{1 \leq j, k \leq p}$. We aim to simultaneously test

$$
H_{0 j}: \mu_{j}=0 \quad \text { versus } \quad H_{1 j}: \mu_{j} \neq 0 \quad \text { for } j=1, \ldots, p .
$$

We are interested in the case where there is strong dependence across the components of $\mathbf{X}$. The approximate factor model assumes that the dependence of a high dimensional random vector $\mathbf{X}$ can be captured by a few factors, that is,

$$
\mathbf{X}=\boldsymbol{\mu}+\mathbf{B f}+\boldsymbol{\Sigma}(\mathbf{f}) \mathbf{u},
$$

from which we observe independent random samples $\left(\mathbf{X}_{1}, \mathbf{f}_{1}\right), \ldots,\left(\mathbf{X}_{n}, \mathbf{f}_{n}\right)$ satisfying

$$
\mathbf{X}_{i}=\left(X_{i 1}, \ldots, X_{i p}\right)^{\top}=\boldsymbol{\mu}+\mathbf{B} \mathbf{f}_{i}+\boldsymbol{\Sigma}\left(\mathbf{f}_{i}\right) \mathbf{u}_{i}, \quad i=1, \ldots, n .
$$

Here, $\mathbf{B}=\left(\boldsymbol{b}_{1}, \ldots, \boldsymbol{b}_{p}\right)^{\top} \in \mathbb{R}^{p \times K}$ represents the factor loading matrix, $\mathbf{f}_{i}$ is the $K$-dimensional common factor to the $i$ th observation and is independent of the idiosyncratic noise $\mathbf{u}_{i} \in \mathbb{R}^{p}$, and $\boldsymbol{\Sigma}(\mathbf{f})=\operatorname{diag}\left(\sigma_{1}(\mathbf{f}), \ldots, \sigma_{p}(\mathbf{f})\right)$ with $\sigma_{j}(\cdot): \mathbb{R}^{K} \mapsto$ $(0, \infty)$ as unknown variance heteroscedasticity functions. We allow the components of $\mathbf{u}_{i}$ to be dependent. To fully understand the influence of heavy tailedness, in this paper we restrict our attention to such an observable factor model.

For testing the hypotheses in (1.5) under model (1.6), when the factor $\mathbf{f}$ is unobserved, a popular and natural approach is based on (marginal) sample averages of $\left\{\mathbf{X}_{i}\right\}$ with a focus on the valid control of FDR [Benjamini and Yekutieli (2001), Genovese and Wasserman (2004), Efron (2007), Sun and Cai (2009), Schwartzman and Lin (2011), Fan and Han (2017)]. As pointed out in Fan, Han and Gu (2012), the power of such an approach is dominated by the factor-adjusted approach that produces alternative rankings of statistical significance from those of the marginal statistics. They focus on a Gaussian model where both $\mathbf{f}$ and $\mathbf{u}$ follow multivariate normal distributions, while statistical properties of the corresponding test procedure on FDR/FDP control remain unclear. The normality assumption, however, is really an idealization which provides insights into the key issues underlying the problems. Data subject to heavy-tailed and asymmetric errors are repeatedly observed in many fields of research [Finkenstadt and Rootzeén (2003)]. For example, it is known that financial returns typically exhibit heavy tails. The important papers by Mandelbrot (1963) and Fama (1963) provide evidence of power-law behavior 

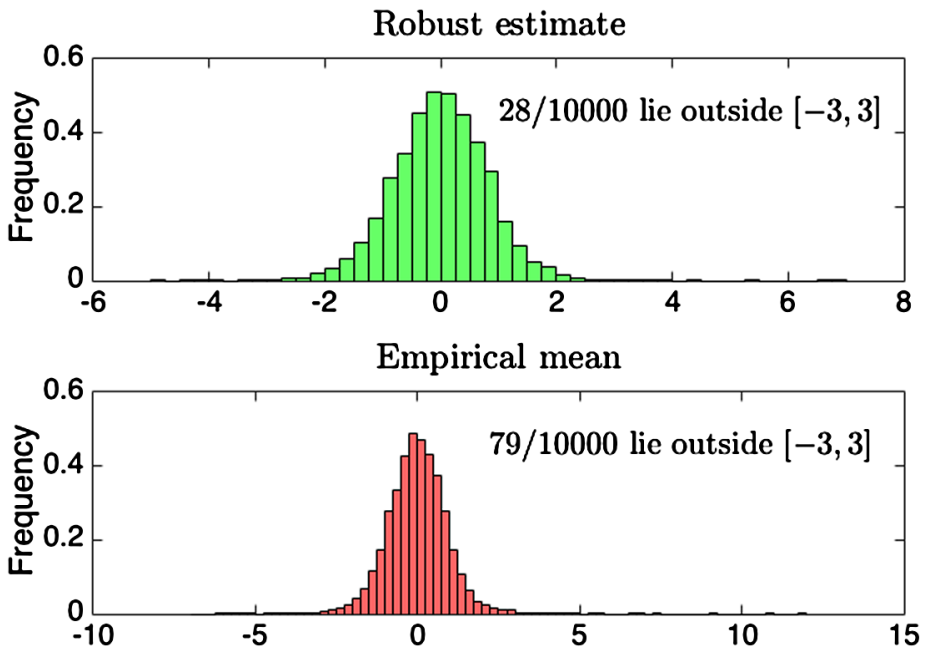

FIG. 1. Histograms of 10,000 robust mean estimates and empirical means based on 30 i.i.d. samples drawn from a normalized $t$-distribution with 2.5 degrees of freedom. Both the mean estimates are rescaled by $\sqrt{30}$.

in asset prices in the early 1960s. Since then, the non-Gaussian character of the distribution of price changes has been widely observed in various market data. Cont (2001) provides further evidence showing that a Student's $t$-distribution with four degrees of freedom displays a tail behavior similar to many asset returns.

For multiple testing with heavy-tailed data, the least squares based test statistics are sensitive to outliers, and thus lack robustness. This issue is amplified further by high dimensionality: When the dimension is large, even moderate tails may lead to significant false discoveries. This motivates us to develop new test statistics that are robust to the tails of error distributions. Also, since the multiple testing problem is more complicated with dependent data, theoretical guarantees of the FDP control for the existing dependence-adjusted methods remain unclear.

To illustrate the impact of heavy tailedness, we generate independent and identically distributed (i.i.d.) random variables $\left\{X_{i j}, i=1, \ldots, 30, j=1, \ldots, 10,000\right\}$ from a normalized $t$-distribution with 2.5 degrees of freedom. In Figure 1, we compare the histogram of the empirical means $\bar{X}_{j}$ with that of the robust mean estimates constructed using (1.3) without covariates, after rescaling both estimators by $\sqrt{30}$. For a standard normal distribution, we expect $99.73 \%$ data points to lie within three standard deviations of the mean or inside $[-3,3]$. Hence for this experiment, if the distribution of the estimator is indeed approximately normal, we would expect about 27 out of 10,000 realizations to lie outside [ $-3,3]$. From Figure 1, we see that the robust procedure gives 28 points that fall outside this interval, whereas the sample average gives a much larger number, 79, many of which would surely be falsely regarded as signals. We see that in the presence of heavy tails and high dimensions, the robust method leads to a more accurate normal tail 
approximation than using a nonrobust one. In fact, for the empirical means, many of them even fall outside $[-6,6]$. This inaccuracy in tail approximation for the nonrobust estimator gives rise to false discoveries. In summary, outliers from the test statistics $\bar{X}_{j}$ can be so large that they are mistakenly regarded as discoveries, whereas the robust approach results in fewer outliers.

In Section 3, we develop robust dependence-adjusted multiple testing procedures with solid theoretical guarantees. We use the approximate factor model (1.6) with an observable factor and relatively heavy-tailed errors to characterize the dependence structure in high dimensional data. Assuming such a model, we construct robust test statistics based on the Huber estimator with a diverging tuning parameter, denoted by $T_{1}, \ldots, T_{p}$, for testing the individual hypotheses. At a prespecified level $0<\alpha<1$, we apply a family of FDP controlling procedures to the dependence-adjusted $P$-values $\left\{P_{j}=2 \Phi\left(-\left|T_{j}\right|\right)\right\}_{j=1}^{p}$ to decide which null hypotheses are rejected, where $\Phi$ is the standard normal distribution function. To justify the validity of the resulting procedure on FDP control, a delicate analysis of the impact of dependence-adjustment on the distribution of the $P$-values is required. We show that, under mild moment and regularity conditions, the robust multiple testing procedure controls the FDP at any prespecified level asymptotically. Specifically, applying Storey's procedure [Storey (2002)] to the above $P$ values gives a data-driven rejection threshold $\widehat{z}_{\mathrm{N}}$ such that $H_{0 j}$ is rejected whenever $\left|T_{j}\right| \geq \widehat{z}_{\mathrm{N}}$. Let $\operatorname{FDP}(z)=V(z) / \max \{1, R(z)\}$ be the FDP at threshold $z \geq 0$, where $V(z)=\sum_{j=1}^{p} 1\left(\left|T_{j}\right| \geq z, \mu_{j}=0\right)$ and $R(z)=\sum_{j=1}^{p} 1\left(\left|T_{j}\right| \geq z\right)$ are the number of false discoveries and the number of total discoveries, respectively. In the ultra-high dimensional setting that $p$ can be as large as $e^{n^{c}}$ for some $0<c<1$, we prove that

$$
\frac{p}{p_{0}} \operatorname{FDP}\left(\widehat{z}_{\mathrm{N}}\right) \rightarrow \alpha \quad \text { in probability }
$$

as $(n, p) \rightarrow \infty$, where $p_{0}=\sum_{j=1}^{p} 1\left(\mu_{j}=0\right)$ is the number of true null hypotheses. We also illustrate the usefulness of the robust techniques by contrasting the performances of robust and least squares based inference procedures through synthetic numerical experiments.

Key technical tools in proving (1.7) are the Berry-Esseen bound and Cramértype moderate deviation for the marginal statistic $T_{j}$. These results are built upon the nonasymptotic Bahadur representation (1.4), and may be of independent interest for other statistical applications. For example, Delaigle, Hall and Jin (2011) explore moderate and large deviations of the $t$-statistic in a variety of high dimensional settings.

1.3. Organization of the paper. The layout of the paper is as follows. In Section 2, we develop a general finite sample theory for Huber's robust $M$-estimator from a new perspective where a diverging tuning parameter is involved. In Section 3 , we propose a robust dependence-adjusted multiple testing procedure with 
rigorous theoretical guarantees. Section 4 consists of numerical studies and real data analysis. The simulation study provides empirical evidence that the proposed robust inference procedure improves performance in the presence of asymmetric and heavy-tailed errors, and maintains efficiency under light-tailed situations. A discussion is given in Section 5. Proofs of the theoretical results in Sections 2 and 3 are provided in the supplemental material [Zhou et al. (2018)].

Notation. For a vector $\mathbf{u}=\left(u_{1}, \ldots, u_{p}\right)^{\top} \in \mathbb{R}^{p}(p \geq 2)$, we use $\|\mathbf{u}\|=$ $\left(\sum_{j=1}^{p} u_{j}^{2}\right)^{1 / 2}$ to denote its $\ell_{2}$-norm. Let $\mathbb{S}^{p-1}=\left\{\mathbf{u} \in \mathbb{R}^{p}:\|\mathbf{u}\|=1\right\}$ represent the unit sphere in $\mathbb{R}^{p}$. For a matrix $\mathbf{A} \in \mathbb{R}^{p \times p},\|\mathbf{A}\|=\sup _{\mathbf{u} \in \mathbb{S}^{p-1}}\|\mathbf{A u}\|$ denotes the spectral norm of $\mathbf{A}$. For any two sequences $\left\{a_{n}\right\}$ and $\left\{b_{n}\right\}$ of positive numbers, denote by $a_{n} \asymp b_{n}$ when $c b_{n} \leq a_{n} \leq C b_{n}$ for some absolute constants $C \geq c>0$, denote by $a_{n} \sim b_{n}$ if $a_{n} / b_{n} \rightarrow 1$ as $n \rightarrow \infty$. Moreover, we write $a_{n}=O\left(b_{n}\right)$ if $a_{n} \leq C b_{n}$ for some absolute constant $C>0$, write $a_{n}=o\left(b_{n}\right)$ if $a_{n} / b_{n} \rightarrow 0$ as $n \rightarrow \infty$, and write $a_{n}=o_{\mathbb{P}}\left(b_{n}\right)$ if $a_{n} / b_{n} \rightarrow 0$ in probability as $n \rightarrow \infty$. For a set $S$, we use $S^{\mathrm{c}}$ to denote its complement and $\operatorname{Card}(S)$ for its cardinality. For $x \in \mathbb{R}$, denote by $\lfloor x\rfloor$ the largest integer not greater than $x$ and $\lceil x\rceil$ the smallest integer not less than $x$. For any two real numbers $a$ and $b$, we write $a \vee b=\max (a, b)$ and $a \wedge b=\min (a, b)$.

2. Robust $\boldsymbol{M}$-estimation: A finite sample theory. Consider a heteroscedastic linear regression model $Y=\mu^{*}+\mathbf{X}^{\top} \boldsymbol{\beta}^{*}+\sigma(\mathbf{X}) \varepsilon$, from which we observe independent samples $\left\{\left(Y_{i}, \mathbf{X}_{i}\right)\right\}_{i=1}^{n}$ satisfying

$$
Y_{i}=\mu^{*}+\mathbf{X}_{i}^{\top} \boldsymbol{\beta}^{*}+\sigma\left(\mathbf{X}_{i}\right) \varepsilon_{i}, \quad i=1, \ldots, n,
$$

where $\mu^{*}$ is the intercept, $\mathbf{X} \in \mathbb{R}^{d}$ is the vector of covariates, $\boldsymbol{\beta}^{*} \in \mathbb{R}^{d}$ is the vector of regression coefficients, $\varepsilon$ is the random error independent of $\mathbf{X}$ and $\sigma(\cdot): \mathbb{R}^{d} \mapsto(0, \infty)$ is an unknown variance function. We assume both $\mathbf{X}$ and $\varepsilon$ have zero means. Under this assumption, $\mu^{*}$ and $\boldsymbol{\beta}^{*}$ together are related to the conditional mean effect of $Y$ given $\mathbf{X}$, and $\mu^{*}$ is the unconditional mean of $Y$ that is of independent interest in many applications. For simplicity, we introduce the following notation:

$$
\begin{aligned}
& \boldsymbol{\theta}^{*}=\left(\mu^{*}, \boldsymbol{\beta}^{* \top}\right)^{\top} \in \mathbb{R}^{d+1}, \quad \mathbf{Z}=\left(1, \mathbf{X}^{\top}\right)^{\top} \in \mathbb{R}^{d+1}, \quad v=\sigma(\mathbf{X}) \varepsilon \quad \text { and } \\
& \mathbf{Z}_{i}=\left(1, \mathbf{X}_{i}^{\top}\right)^{\top}, \quad v_{i}=\sigma\left(\mathbf{X}_{i}\right) \varepsilon_{i}, \quad i=1, \ldots, n .
\end{aligned}
$$

In this section, we study the robust estimator of $\boldsymbol{\theta}^{*}$ defined in (1.2). In particular, we show that it admits an exponential-type deviation bound even for heavy-tailed error distributions. Note that, under the heteroscedastic model $(2.1), \boldsymbol{\theta}^{*}$ differs from the median effect of $Y$ conditioning on $\mathbf{X}$, so that the LAD-based methods are not applicable to estimate $\boldsymbol{\theta}^{*}$. Instead, we focus on Huber's robust estimator $\widehat{\boldsymbol{\theta}}$ given in (1.2) with a diverging tuning parameter $\tau=\tau_{n}$ that balances the approximation error and robustness of the estimator. To begin with, we make the following conditions on the linear model (2.1). 
Condition 2.1. (i) The random vector $\mathbf{X} \in \mathbb{R}^{d}$ satisfies $\mathbb{E}(\mathbf{X})=\mathbf{0}$, $\mathbb{E}\left(\mathbf{X X}^{\top}\right)=\boldsymbol{\Sigma}$ for some positive definite matrix $\boldsymbol{\Sigma}$ and $K_{0}:=\left\|\boldsymbol{\Sigma}^{-1 / 2} \mathbf{X}\right\|_{\psi_{2}}<\infty$, where $\|\cdot\|_{\psi_{2}}$ denotes the vector sub-Gaussian norm [Vershynin (2012)]. (ii) Independent of $\mathbf{X}$, the error variable $\varepsilon$ satisfies $\mathbb{E}(\varepsilon)=0$ and $\mathbb{E}\left(\varepsilon^{2}\right)=1$. (iii) $\sigma(\cdot)$ : $\mathbb{R}^{d} \mapsto(0, \infty)$ is a positive function and $\sigma^{2}:=\mathbb{E}\left\{\sigma^{2}(\mathbf{X})\right\}$ is finite.

Condition 2.1 allows a family of conditional heteroscedastic models with heavytailed error $\varepsilon$. Specifically, it only requires the second moment of $v=\sigma(\mathbf{X}) \varepsilon$ to be finite. Under this condition, our first result, Theorem 2.1, provides an exponentialtype deviation bound and a nonasymptotic Bahadur representation for the robust estimator $\widehat{\boldsymbol{\theta}}=\left(\widehat{\mu}, \widehat{\boldsymbol{\beta}}^{\mathrm{T}}\right)^{\mathrm{T}}$ defined in (1.2).

THEOREM 2.1. Under the linear model (2.1) with Condition 2.1 satisfied, we have for any $w>0$ that, the robust estimator $\widehat{\boldsymbol{\theta}}$ in (1.2) with $\tau=\tau_{n}=\tau_{0} \sqrt{n}(d+$ $1+w)^{-1 / 2}$ and $\tau_{0} \geq \sigma$ satisfies

$$
\begin{array}{r}
\mathbb{P}\left\{\left\|\mathbf{S}^{1 / 2}\left(\widehat{\boldsymbol{\theta}}-\boldsymbol{\theta}^{*}\right)\right\|>a_{1}(d+w)^{1 / 2} n^{-1 / 2}\right\} \leq 7 e^{-w} \quad \text { and } \\
\mathbb{P}\left\{\left\|\mathbf{S}^{1 / 2}\left(\widehat{\boldsymbol{\theta}}-\boldsymbol{\theta}^{*}\right)-\frac{1}{n} \sum_{i=1}^{n} \ell_{\tau}^{\prime}\left(v_{i}\right) \mathbf{S}^{-1 / 2} \mathbf{Z}_{i}\right\|>a_{2} \frac{d+w}{n}\right\} \leq 8 e^{-w}
\end{array}
$$

as long as $n \geq a_{3}(d+w)^{3 / 2}$, where $\mathbf{S}=\mathbb{E}\left(\mathbf{Z Z}^{\top}\right)$ and $a_{1}-a_{3}$ are positive constants depending only on $\tau_{0}, K_{0}$ and $\left\|\mathbf{S}^{-1 / 2} \overline{\mathbf{S}} \mathbf{S}^{-1 / 2}\right\|$ with $\overline{\mathbf{S}}=\mathbb{E}\left\{\sigma^{2}(\mathbf{X}) \mathbf{Z Z} \mathbf{Z}^{\top}\right\}$.

An important message of Theorem 2.1 is that, even for heavy-tailed errors with only finite second moment, the robust estimator $\widehat{\boldsymbol{\theta}}$ with properly chosen $\tau$ has sub-Gaussian tails. See inequality (2.2). To some extent, the tuning parameter $\tau$ plays a similar role as the bandwidth in constructing nonparametric estimators. Furthermore, we show in (2.3) that the remainder of the Bahadur representation for $\widehat{\boldsymbol{\theta}}$ exhibits sub-exponential tails. To the best of our knowledge, no nonasymptotic results of this type exist in the literature, and classical asymptotic results can only be used to derive polynomial-type deviation bounds.

Write $W_{n}:=n^{-1 / 2} \sum_{i=1}^{n} \ell_{\tau}^{\prime}\left(v_{i}\right)$. As a direct consequence of $(2.3), \sqrt{n}\left(\widehat{\mu}-\mu^{*}\right)$ is close to $W_{n}$ with probability approaching one exponentially fast. The next result shows that, under higher moment condition on $v=\sigma(\mathbf{X}) \varepsilon, W_{n}$ has an asymptotic normal distribution with mean 0 and variance $\sigma^{2}=\mathbb{E}\left(v^{2}\right)$.

THEOREM 2.2. Assume Condition 2.1 holds and $v_{\kappa}:=\mathbb{E}\left(|v|^{\kappa}\right)$ is finite for some $\kappa \geq 3$. Then there exists an absolute constant $C>0$ such that for any $\tau>0$,

$$
\begin{aligned}
\sup _{x \in \mathbb{R}} \mid \mathbb{P} & \left(\sigma^{-1} W_{n} \leq x\right)-\Phi(x) \mid \\
& \leq C\left(\sigma^{-3} v_{3} n^{-1 / 2}+\sigma^{-2} v_{\kappa} \tau^{2-\kappa}+\sigma^{2} \tau^{-2}+\sigma^{-1} v_{\kappa} \tau^{1-\kappa} \sqrt{n}\right) .
\end{aligned}
$$


In particular, we have

$$
\sup _{x \in \mathbb{R}}\left|\mathbb{P}\left(\sigma^{-1} W_{n} \leq x\right)-\Phi(x)\right| \leq C\left(\sigma^{-3} v_{3} n^{-1 / 2}+\sigma^{-2} v_{4} \tau^{-2}+\sigma^{-1} v_{4} \tau^{-3} \sqrt{n}\right) .
$$

Together, Theorems 2.1 and 2.2 lead to a Berry-Esseen-type bound for $T:=$ $\sqrt{n}\left(\widehat{\mu}-\mu^{*}\right) / \sigma$ for properly chosen $\tau$. In addition, the following theorem gives a Cramér-type moderation deviation result for $T$, which quantifies the relative error of the normal approximation.

THEOREM 2.3. Assume Condition 2.1 is met, $\mathbb{E}\left(|v|^{3}\right)<\infty$ and let $\left\{w_{n}\right\}_{n \geq 1}$ be an arbitrary sequence of positive numbers satisfying $w_{n} \rightarrow \infty$ and $w_{n}=o(\sqrt{n})$. Then the statistic $T$ with $\tau=\tau_{0} \sqrt{n}\left(d+w_{n}\right)^{-1 / 2}$ for some constant $\tau_{0} \geq \sigma$ satisfies

$$
\mathbb{P}(|T| \geq z)=\left(1+C_{n, z}\right) \mathbb{P}(|G| \geq z)
$$

uniformly for $0 \leq z=o\left\{\min \left(\sqrt{w_{n}}, \sqrt{n} w_{n}^{-1}\right)\right\}$ as $n \rightarrow \infty$, where $G \sim N(0,1)$,

$$
\left|C_{n, z}\right| \leq C\left\{(\sqrt{\log n}+z)^{3} n^{-1 / 2}+(1+z)\left(n^{-3 / 10}+n^{-1 / 2} w_{n}\right)+e^{-w_{n}}\right\}
$$

and $C>0$ is a constant independent of $n$. In particular, we have

$$
\sup _{0 \leq z \leq o\left\{\min \left(\sqrt{w_{n}}, \sqrt{n} w_{n}^{-1}\right)\right\}}\left|\frac{\mathbb{P}(|T| \geq z)}{2-2 \Phi(z)}-1\right| \rightarrow 0 .
$$

REMARK 2.1. From Theorem 2.3, we see that the ratio $\mathbb{P}(|T| \geq z) /\{2-$ $2 \Phi(z)\}$ is close to 1 for a wide range of nonnegative $z$-values, whose length depends on both the sample size $n$ and $w_{n}$. In particular, taking $w_{n} \asymp n^{1 / 3}$ gives the widest possible range $\left[0, o\left(n^{1 / 6}\right)\right)$, which is also optimal for Cramér-type moderate deviation results [Linnik (1961)]. In this case, the tuning parameter $\tau=\tau_{n}$ is of order $n^{1 / 3}$.

REMARK 2.2. Motivated by an application to large-scale simultaneous inference considered in Section 3, we only focus on the robust intercept estimator $\widehat{\mu}$ of $\mu^{*}$ in Theorems 2.2 and 2.3. In fact, similar results can be obtained for $\widehat{\boldsymbol{\beta}}$ or a specific coordinate of $\widehat{\boldsymbol{\beta}}$ based on the Bahadur representation (2.3).

3. Large-scale multiple testing for heavy-tailed dependent data. In this section, we propose and analyze a robust dependence-adjusted procedure for simultaneously testing the means $\mu_{1}, \ldots, \mu_{p}$ in model (1.6), based on independent observations from the population vector $\mathbf{X}$ which exhibits strong dependence and heavy tails. 
3.1. Robust test statistics. Suppose we are given independent random samples $\left\{\left(\mathbf{X}_{i}, \mathbf{f}_{i}\right)\right\}_{i=1}^{n}$ from model (1.6). We are interested in the simultaneous testing of mean effects (1.5). A naive approach under normality is to directly use the information $X_{i j} \sim N\left(\mu_{j}, \sigma_{j j}\right)$ for the dependent case as was done in the literature, where $\sigma_{j j}=\operatorname{var}\left(X_{j}\right)$. Such an approach is very natural and popular when the factors are unobservable and focus is on the valid control of FDR, but is inefficient as noted in Fan, Han and Gu (2012). Indeed, if the loading matrix $\mathbf{B}$ is known and the factors are observed (otherwise, replaced by their estimates), for each $j$, we can construct the marginal test statistic using dependence-adjusted observations $\left\{X_{i j}-\boldsymbol{b}_{j}^{\top} \mathbf{f}_{i}\right\}_{i=1}^{n}$ from $\mu_{j}+\sigma_{j}(\mathbf{f}) u_{j}$ for testing the $j$ th hypothesis $H_{0 j}: \mu_{j}=0$.

We consider the approximate factor model (1.6) and write

$$
\begin{aligned}
\mathbf{u} & =\left(u_{1}, \ldots, u_{p}\right)^{\top}, \quad \boldsymbol{v}=\left(v_{1}, \ldots, v_{p}\right)^{\top}=\boldsymbol{\Sigma}(\mathbf{f}) \mathbf{u}, \\
\boldsymbol{v}_{i} & =\left(v_{i 1}, \ldots, v_{i p}\right)^{\top}=\boldsymbol{\Sigma}\left(\mathbf{f}_{i}\right) \mathbf{u}_{i}, \quad i=1, \ldots, n .
\end{aligned}
$$

Let $\boldsymbol{\Sigma}_{f}$ and $\boldsymbol{\Sigma}_{v}=\left(\sigma_{v, j k}\right)_{1 \leq j, k \leq p}$ denote the covariance matrices of $\mathbf{f}$ and $\boldsymbol{v}$, respectively. Under certain sparsity condition on $\boldsymbol{\Sigma}_{v}$ (see Section 3.4 for an elaboration), $v_{1}, \ldots, v_{p}$ are weakly dependent random variables with higher signal-to-noise ratios since $\operatorname{var}\left(v_{j}\right)=\sigma_{j j}-\left\|\Sigma_{f}^{1 / 2} \boldsymbol{b}_{j}\right\|^{2}<\sigma_{j j}$. Therefore, subtracting common factors out makes the resulting FDP control procedure more efficient and powerful. It provides an alternative ranking of the significance of hypothesis from the tests based on marginal statistics.

For each $j=1, \ldots, p$, we have a linear regression model:

$$
X_{i j}=\mu_{j}+\boldsymbol{b}_{j}^{\top} \mathbf{f}_{i}+v_{i j}, \quad i=1, \ldots, n .
$$

A natural approach is to estimate $\mu_{j}$ and $\boldsymbol{b}_{j}$ by the method of least squares. However, the least squares method is sensitive to the tails of the error distributions. Also, as noted in Fan, Li and Wang (2017), the LAD-based methods are not applicable in the presence of asymmetric and heteroscedastic errors. Hence, we suggest a robust method that simultaneously estimates $\mu_{j}$ and $\boldsymbol{b}_{j}$ by solving

$$
\left(\widehat{\mu}_{j}, \widehat{\boldsymbol{b}}_{j}^{\top}\right)^{\top} \in \underset{\mu \in \mathbb{R}, \boldsymbol{b} \in \mathbb{R}^{d}}{\operatorname{argmin}} \sum_{i=1}^{n} \ell_{\tau}\left(X_{i j}-\mu-\boldsymbol{b}^{\top} \mathbf{f}_{i}\right),
$$

where $\ell_{\tau}$ is given in (1.1). By Theorems 2.1 and 2.2, the adaptive Huber estimator $\widehat{\mu}_{j}$ follows a normal distribution asymptotically as $n \rightarrow \infty$ :

$$
\sqrt{n}\left(\widehat{\mu}_{j}-\mu_{j}\right) \stackrel{\mathscr{D}}{\rightarrow} N\left(0, \sigma_{\nu, j j}\right) \quad \text { with } \sigma_{\nu, j j}=\operatorname{var}\left(v_{j}\right) .
$$

To construct a test statistic for the individual hypothesis $H_{0 j}: \mu_{j}=0$ with pivotal limiting distribution, we need to estimate $\sigma_{v, j j}=\sigma_{j j}-\operatorname{var}\left(\boldsymbol{b}_{j}^{\top} \mathbf{f}\right)$. For $\operatorname{var}\left(\boldsymbol{b}_{j}^{\top} \mathbf{f}\right)$, a natural and simple estimator is $\widehat{\boldsymbol{b}}_{j}^{\top} \widehat{\boldsymbol{\Sigma}}_{f} \widehat{\boldsymbol{b}}_{j}$, where $\widehat{\boldsymbol{\Sigma}}_{f}:=n^{-1} \sum_{i=1}^{n} \mathbf{f}_{i} \mathbf{f}_{i}^{\top}$. Let $\widehat{\sigma}_{j j}$ and 
$\widehat{\sigma}_{v, j j}$ be generic estimators of $\sigma_{j j}$ and $\sigma_{v, j j}$, respectively. To simultaneously infer all the hypotheses of interest, we need the following uniform convergence results:

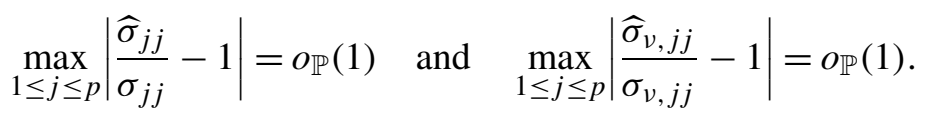

For $\sigma_{j j}=\operatorname{var}\left(X_{j}\right)$, it is known that the sample variance $n^{-1} \sum_{i=1}^{n}\left(X_{i j}-\bar{X}_{j}\right)^{2}$ performs poorly when $X_{j}$ has heavy tails. Based on the recent developments of robust mean estimation for heavy-tailed data [Catoni (2012), Joly and Lugosi (2016), Fan, Li and Wang (2017)], we consider the following two types of robust variance estimators:

1. (Adaptive Huber variance estimator). Write $\theta_{j}=\mathbb{E}\left(X_{j}^{2}\right)$ so that $\sigma_{j j}=\theta_{j}-$ $\mu_{j}^{2}$. Construct the adaptive Huber estimator of $\theta_{j}$ using the squared data, that is, $\widehat{\theta}_{j}=\operatorname{argmin}_{\theta>0} \sum_{i=1}^{n} \ell_{\gamma}\left(X_{i j}^{2}-\theta\right)$, where $\gamma=\gamma_{n}$ is a tuning parameter. Then we compute the adaptive Huber estimator $\left(\widehat{\mu}_{j}, \widehat{\boldsymbol{b}}_{j}^{\top}\right)^{\top}$ given in (3.2). The variance estimator is then defined by

$$
\widehat{\sigma}_{v, j j}= \begin{cases}\widehat{\theta}_{j}-\widehat{\mu}_{j}^{2}-\widehat{\boldsymbol{b}}_{j}^{\top} \widehat{\boldsymbol{\Sigma}}_{f} \widehat{\boldsymbol{b}}_{j}, & \text { if } \widehat{\theta}_{j}>\widehat{\mu}_{j}^{2}+\widehat{\boldsymbol{b}}_{j}^{\top} \widehat{\boldsymbol{\Sigma}}_{f} \widehat{\boldsymbol{b}}_{j}, \\ \text { otherwise. }\end{cases}
$$

2. (Median-of-means variance estimator). The median-of-means technique, which dates back to Nemirovsky and Yudin (1983), robustifies the empirical mean by first dividing the given observations into several blocks, computing the sample mean within each block and then taking the median of these sample means as the final estimator. Although the sample variance cannot be represented in a simple average form, it is a $U$-statistic with a symmetric kernel $h: \mathbb{R}^{2} \mapsto \mathbb{R}$ given by $h(x, y)=(x-y)^{2} / 2$. The recent work of Joly and Lugosi (2016) extends the median-of-means technique to construct $U$-statistics based sub-Gaussian estimators for heavy-tailed data.

Back to the current problem, we aim to estimate $\sigma_{j j}$ based on independent observations $X_{1 j}, \ldots, X_{n j}$. Let $V=V_{n}<n$ be an integer and decompose $n$ as $n=V m+r$ for some integer $0 \leq r<V$. Let $B_{1}, \ldots, B_{V}$ be a partition of $\{1, \ldots, n\}$ defined by

$$
B_{k}= \begin{cases}\{(k-1) m+1,(k-1) m+2, \ldots, k m\} & \text { if } 1 \leq k \leq V-1, \\ \{(V-1) m+1,(V-1) m+2, \ldots, n\} & \text { if } k=V .\end{cases}
$$

For each pair $(k, \ell)$ satisfying $1 \leq k<\ell \leq V$, define decoupled $U$-statistic $U_{j, k \ell}=$ $\left(2\left|B_{k}\right|\left|B_{\ell}\right|\right)^{-1} \sum_{i_{1} \in B_{k}} \sum_{i_{2} \in B_{\ell}}\left(X_{i_{1} j}-X_{i_{2} j}\right)^{2}$. Then we estimate $\sigma_{j j}$ by the median of $\left\{U_{j, k \ell}: 1 \leq k<\ell \leq V\right\}$, that is, $\widetilde{\sigma}_{j j}(V) \in \operatorname{argmin}_{u \in \mathbb{R}} \sum_{1 \leq k<\ell \leq V}\left|U_{j, k \ell}-u\right|$. As before, we compute the adaptive Huber estimator $\left(\widehat{\mu}_{j}, \widehat{\boldsymbol{b}}_{j}^{\top}\right)^{\top}$. Finally, our robust variance estimators are

$$
\widetilde{\sigma}_{v, j j}= \begin{cases}\widetilde{\sigma}_{j j}(V)-\widehat{\boldsymbol{b}}_{j}^{\top} \widehat{\boldsymbol{\Sigma}}_{f} \widehat{\boldsymbol{b}}_{j} & \text { if } \widetilde{\sigma}_{j j}(V)>\widehat{\boldsymbol{b}}_{j}^{\top} \widehat{\boldsymbol{\Sigma}}_{f} \widehat{\boldsymbol{b}}_{j}, \\ \tilde{\sigma}_{j j}(V) & \text { otherwise. }\end{cases}
$$


Given robust mean and variance estimators of each type, we construct dependence-adjusted test statistics:

$$
T_{j}=\sqrt{n} \widehat{\sigma}_{v, j j}^{-1 / 2} \widehat{\mu}_{j} \quad \text { and } \quad S_{j}=\sqrt{n} \widetilde{\sigma}_{v, j j}^{-1 / 2} \widehat{\mu}_{j} \quad \text { for } j=1, \ldots, p .
$$

In fact, as long as the fourth moment $\mathbb{E}\left(X_{j}^{4}\right)$ is finite, the estimators $\widehat{\sigma}_{\nu, j j}$ and $\tilde{\sigma}_{\nu, j j}$ given in (3.4) and (3.6), respectively, are concentrated around $\sigma_{\nu, j j}$ with high probability. In view of (3.3), under the null hypothesis $H_{0 j}: \mu_{j}=0$, the adjusted test statistics $T_{j}$ and $S_{j}$ satisfy that $T_{j} \stackrel{\mathscr{D}}{\rightarrow} N(0,1)$ and $S_{j} \stackrel{\mathscr{D}}{\rightarrow} N(0,1)$ as $n \rightarrow \infty$.

3.2. Dependence-adjusted FDP control procedure. To conduct multiple testing of (1.5) using the test statistics $T_{j}$ 's, let $z>0$ be the critical value to be determined such that $H_{0 j}$ is rejected whenever $\left|T_{j}\right| \geq z$. The main object of interest in this paper is the false discovery proportion

$$
\operatorname{FDP}(z)=\frac{V(z)}{\max \{R(z), 1\}}, \quad z \geq 0,
$$

where $V(z)=\sum_{j \in \mathcal{H}_{0}} 1\left(\left|T_{j}\right| \geq z\right)$ is the number of false discoveries, $R(z)=$ $\sum_{j=1}^{p} 1\left(\left|T_{j}\right| \geq z\right)$ and $\mathcal{H}_{0}=\left\{j: 1 \leq j \leq p, \mu_{j}=0\right\}$ represents the set of true null hypotheses. There is substantial interest in controlling the FDP at a prespecified level $0<\alpha<1$ for which the ideal rejection threshold is $z_{\text {oracle }}=\inf \{z \geq 0$ : $\operatorname{FDP}(z) \leq \alpha\}$

The statistical behavior of $\operatorname{FDP}(z)$ is the center of interest in multiple testing. However, the realization of $V(z)$ for a given experiment is unknown, and thus needs to be estimated. When the sample size is large, it is natural to approximate $V(z)$ by its expectation $2 p_{0} \Phi(-z)$, where $p_{0}=\operatorname{Card}\left(\mathcal{H}_{0}\right)$. In the high dimensional sparse setting, both $p$ and $p_{0}$ are large and $p_{1}=p-p_{0}=o(p)$ is relatively small. Therefore, we can use $p$ as a slightly conservative surrogate for $p_{0}$, so that $\operatorname{FDP}(z)$ can be approximated by

$$
\operatorname{FDP}_{\mathrm{N}}(z)=\frac{2 p \Phi(-z)}{\max \{R(z), 1\}}, \quad z \geq 0 .
$$

We will prove in Theorem 3.2 that under mild conditions, $\operatorname{FDP}_{\mathrm{N}}(z)$ provides a consistent estimate of $\operatorname{FDP}(z)$ uniformly in $0 \leq z \leq \Phi^{-1}\left(1-m_{p} /(2 p)\right)$ for any sequence of positive number $m_{p} \leq 2 p$ satisfying $m_{p} \rightarrow \infty$.

In the nonsparse case where $\pi_{0}=p_{0} / p$ is bounded away from 0 and 1 as $p \rightarrow \infty, \mathrm{FDP}_{\mathrm{N}}$ given in (3.9) tends to overestimate the true FDP. Therefore, we need to estimate the proportion $\pi_{0}$, which has been studied by Efron et al. (2001), Storey (2002), Genovese and Wasserman (2004), Langaas, Lindqvist and Ferkingstad (2005) and Meinshausen and Rice (2006), among others. For simplicity, we focus on Storey's approach. Let $\left\{P_{j}=2 \Phi\left(-\left|T_{j}\right|\right)\right\}_{j=1}^{p}$ be the approximate 
$P$-values. For a predetermined $\lambda \in[0,1)$, Storey (2002) suggests the following conservative estimate of $\pi_{0}$ :

$$
\widehat{\pi}_{0}(\lambda)=\frac{1}{(1-\lambda) p} \sum_{j=1}^{p} 1\left(P_{j}>\lambda\right) .
$$

The intuition of such an estimator is as follows. Since most of the large $P$-values correspond to the null, and thus are uniformly distributed, for a sufficiently large $\lambda$, we expect about $(1-\lambda) \pi_{0}$ of the $P$-values to lie in $(\lambda, 1]$. Hence, the proportion of $P$-values that exceed $\lambda, p^{-1} \sum_{j=1}^{p} 1\left(P_{j}>\lambda\right)$, should be close to $(1-\lambda) \pi_{0}$. This gives rise to Storey's procedure.

Incorporating such an estimate of $\pi_{0}$, we obtain a modified estimate of $\operatorname{FDP}(z)$ by

$$
\operatorname{FDP}_{\mathrm{N}, \lambda}(z)=\frac{2 p \widehat{\pi}_{0}(\lambda) \Phi(-z)}{\max \{R(z), 1\}}, \quad z \geq 0 .
$$

In view of (3.9)-(3.11) and the fact $\widehat{\pi}_{0}(0)=1$, we have $\operatorname{FDP}_{\mathrm{N}, 0}(z)=\operatorname{FDP}_{\mathrm{N}}(z)$.

By replacing the unknown quantity $\operatorname{FDP}(z)$ by $\operatorname{FDP}_{\mathrm{N}, \lambda}(z)$ given in (3.11) for some $\lambda \in[0,1)$, we reject $H_{0 j}$ whenever $\left|T_{j}\right| \geq \widehat{z}_{\mathrm{N}, \lambda}$, where

$$
\widehat{z}_{\mathrm{N}, \lambda}=\inf \left\{z \geq 0: \operatorname{FDP}_{\mathrm{N}, \lambda}(z) \leq \alpha\right\} .
$$

By Lemmas 1 and 2 in Storey, Taylor and Siegmund (2004), this procedure is equivalent to a variant of the seminal Benjamini-Hochberg $(\mathrm{B}-\mathrm{H})$ procedure [Benjamini and Hochberg (1995)] for selecting $\mathcal{S}=\left\{j: 1 \leq j \leq p, P_{j} \leq\right.$ $\left.P_{\left(k_{p}(\lambda)\right)}\right\}$ based on the $P$-values $P_{j}=2 \Phi\left(-\left|T_{j}\right|\right)$, where $k_{p}(\lambda):=\max \{j: 1 \leq$ $\left.j \leq p, P_{(j)} \leq \frac{\alpha j}{\hat{\pi}_{0}(\lambda) p}\right\}$ and $P_{(1)} \leq \cdots \leq P_{(p)}$ are the ordered $P$-values. Theorem 3.3 shows that under weak moment conditions, the FDP of this dependence-adjusted procedure with $\lambda=0$ converges to $\alpha$ in the ultra-high dimensional sparse setting.

Note that $\operatorname{FDP}_{\mathrm{N}, 0}(z)$ is the most conservatively biased estimate of $\operatorname{FDP}(z)$ among all $\lambda \in[0,1)$ using normal calibration. The statistical power of the corresponding procedure can be compromised if $\pi_{0}$ is much smaller than 1 . In general, the procedure requires the choice of a tuning parameter $\lambda$ in the estimate $\widehat{\pi}(\lambda)$, which leads to an inherent bias-variance trade-off. We refer to Section 9 in Storey (2002) and Section 6 in Storey, Taylor and Siegmund (2004) for two data-driven methods for automatically choosing $\lambda$.

3.3. Bootstrap calibration. When the sample size is large, it is suitable to use the normal distribution for calibration. Here, we consider bootstrap calibration, which has been widely used due to its good numerical performance when the sample size is relatively small. In particular, we focus on the weighted bootstrap [Barbe and Bertail (1995)], which perturbs the objective function of an $M$-estimator with i.i.d. weights. Let $W$ be a random variable with unit mean and variance, that is, $\mathbb{E}(W)=1$ and $\operatorname{var}(W)=1$. Independent of $\mathbf{X}_{1}, \ldots, \mathbf{X}_{n}$, generate i.i.d. random 
weights $\left\{W_{i j, b}, 1 \leq i \leq n, 1 \leq j \leq p, 1 \leq b \leq B\right\}$ from $W$, where $B$ is the number of bootstrap replications. For each $j$, the bootstrap counterparts of $\left(\widehat{\mu}_{j}, \widehat{\boldsymbol{b}}_{j}^{\top}\right)^{\top}$ given in (3.2) are defined by

$$
\left(\widehat{\mu}_{j, b}^{*},\left(\widehat{\boldsymbol{b}}_{j, b}^{*}\right)^{\top}\right)^{\top} \in \underset{\mu \in \mathbb{R}, \boldsymbol{b} \in \mathbb{R}^{d}}{\operatorname{argmin}} \sum_{i=1}^{n} W_{i j, b} \ell_{\tau}\left(X_{i j}-\mu-\boldsymbol{b}^{\top} \mathbf{f}_{i}\right), \quad b=1, \ldots, B .
$$

For $j=1, \ldots, p$, define empirical tail distributions

$$
G_{j, B}^{*}(z)=\frac{1}{B+1} \sum_{b=1}^{B} 1\left(\left|\widehat{\mu}_{j, b}^{*}-\widehat{\mu}_{j}\right| \geq z\right), \quad z \geq 0 .
$$

The bootstrap $P$-values are thus given by $\left\{P_{j}^{*}=G_{j, B}^{*}\left(\left|\widehat{\mu}_{j}\right|\right)\right\}_{j=1}^{p}$, to which either the B-H procedure or Storey's procedure can be applied. For the former, we reject $H_{0 j}$ whenever $P_{j}^{*} \leq P_{\left(k_{p}^{*}\right)}^{*}$, where $k_{p}^{*}=\max \left\{j: 1 \leq j \leq p, P_{(j)}^{*} \leq j \alpha / p\right\}$ for a predetermined $0<\alpha<1$ and $P_{(1)}^{*} \leq \cdots \leq P_{(p)}^{*}$ are the ordered bootstrap $P$-values. For the distribution of the bootstrap weights, it is common to choose $W \sim 2$ Bernoulli(0.5), $W \sim \exp (1)$ or $W \sim N(1,1)$ in practice. Using nonnegative random weights has the advantage that the weighted objective function is guaranteed to be convex.

REMARK 3.1. The weighted bootstrap procedure serves as an alternative method to normal calibration in multiple testing. We refer to Spokoiny and Zhilova (2015) and Zhilova (2016) for the most advanced recent results of weighted bootstrap and a comprehensive literature review. We leave the theoretical guarantee of this procedure for future research.

3.4. Theoretical properties. First, we impose some conditions on the distribution of $\mathbf{X}$ and the tuning parameters $\tau$ and $\gamma$ that are used in the robust regression and robust estimation of the second moment:

(C1) (i) $\mathbf{X} \in \mathbb{R}^{p}$ follows the model (1.6) with $\mathbf{f}$ and $\mathbf{u}$ being independent; (ii) $\mathbb{E}\left(u_{j}\right)=0, \mathbb{E}\left(u_{j}^{2}\right)=1$ for $j=1, \ldots, p$, and $c_{v} \leq \min _{1 \leq j \leq p} \sigma_{v, j j} \leq$ $\max _{1 \leq j \leq p} \mathbb{E}\left(v_{j}^{4}\right) \leq C_{v}$ for some $C_{v}>c_{v}>0$; (iii) $\mathbb{E}(\mathbf{f})=\mathbf{0}, \boldsymbol{\Sigma}_{f}=\operatorname{cov}(\mathbf{f})$ is positive definite and $\left\|\Sigma_{f}^{-1 / 2} \mathbf{f}\right\|_{\psi_{2}} \leq C_{f}$ for some $C_{f}>0$.

(C2) $(\tau, \gamma)=\left(\tau_{n}, \gamma_{n}\right)$ satisfies $\tau=\tau_{0} \sqrt{n} w_{n}^{-1 / 2}$ and $\gamma=\gamma_{0} \sqrt{n} w_{n}^{-1 / 2}$ for some constants $\tau_{0} \geq \max _{1 \leq j \leq p} \sigma_{\nu, j j}^{1 / 2}$ and $\gamma_{0} \geq \max _{1 \leq j \leq p} \operatorname{var}^{1 / 2}\left(X_{j}^{2}\right)$, where the sequence $w_{n}$ is such that $w_{n} \rightarrow \infty$ and $w_{n}=o(\sqrt{n})$.

In addition, we need the following assumptions on the covariance structure of $\boldsymbol{v}=\boldsymbol{\Sigma}(\mathbf{f}) \mathbf{u}$, and the number and magnitudes of the signals (nonzero coordinates of $\boldsymbol{\mu})$. Let $\mathbf{R}_{v}=\left(\rho_{v, j k}\right)_{1 \leq j, k \leq p}$ be the correlation matrix of $\boldsymbol{v}$, where by the independence of $\mathbf{f}$ and $\mathbf{u}, \rho_{\nu, j k}=\frac{\mathbb{E}\left\{\sigma_{j}(\mathbf{f}) \sigma_{k}(\mathbf{f})\right\}}{\sqrt{\mathbb{E} \sigma_{j}^{2}(\mathbf{f}) \mathbb{E} \sigma_{k}^{2}(\mathbf{f})}} \times \operatorname{corr}\left(u_{j}, u_{k}\right)$. 
(C3) $\max _{1 \leq j<k \leq p}\left|\rho_{\nu, j k}\right| \leq \rho$ and

$$
s_{p}:=\max _{1 \leq j \leq p} \sum_{k=1}^{p} 1\left\{\left|\rho_{\nu, j k}\right|>(\log p)^{-2-\kappa}\right\}=O\left(p^{r}\right)
$$

for some $0<\rho<1, \kappa>0$ and $0<r<(1-\rho) /(1+\rho)$. As $n, p \rightarrow \infty, p_{0} / p \rightarrow$ $\pi_{0} \in(0,1], \log p=o\left(n^{1 / 5}\right)$ and $w_{n} \asymp n^{1 / 5}$, where $w_{n}$ is as in Condition (C2).

(C4) $\operatorname{Card}\left\{j: 1 \leq j \leq p, \sigma_{v, j j}^{-1 / 2}\left|\mu_{j}\right| \geq \lambda \sqrt{(\log p) / n}\right\} \rightarrow \infty$ as $n, p \rightarrow \infty$ for some $\lambda>2 \sqrt{2}$.

Condition (C3) allows weak dependence among $v_{1}, \ldots, v_{p}$ in the sense that each variable is moderately correlated with $s_{p}$ other variables and weakly correlated with the remaining ones. The technical assumption (C4) imposes a constraint on the number of significant true alternatives, which is slightly stronger than $p_{1} \rightarrow \infty$. According to Proposition 2.1 in Liu and Shao (2014), this condition is nearly optimal for the results on FDP control in the sense that if $p_{1}$ is fixed, the $\mathrm{B}-\mathrm{H}$ method fails to control the FDP at any level $0<\beta<1$ with overwhelming probability even if the true $P$-values were known.

For robust test statistics $T_{j}$ 's given in (3.7), define the null distribution $F_{j, n}(x)=\mathbb{P}\left(T_{j} \leq x \mid H_{0 j}\right)$ and the corresponding $P$-value $P_{j}^{\text {true }}=F_{j, n}\left(-\left|T_{j}\right|\right)+$ $1-F_{j, n}\left(\left|T_{j}\right|\right)$. In practice, we use $P_{j}=2 \Phi\left(-\left|T_{j}\right|\right)$ to estimate the true (unknown) $P$-values. A natural question is on how fast $p$ can diverge with $n$ so as to maintain valid simultaneous inference. This problem has been studied in Fan, Hall and Yao (2007), Kosorok and Ma (2007) and Liu and Shao (2010). There it is shown that the simple consistency $\max _{1 \leq j \leq p}\left|P_{j}-P_{j}^{\text {true }}\right|=o(1)$ is not enough, and the level of accuracy required must increase with $n$. More precisely, to secure a valid inference, we require

$$
\max _{1 \leq j \leq p}\left|\frac{P_{j}}{P_{j}^{\text {true }}}-1\right| 1\left\{\mathcal{S}_{j}\right\}=o(1) \quad \text { as } n \rightarrow \infty,
$$

where $\mathcal{S}_{j}=\left\{P_{j}^{\text {true }}>\alpha / p\right\}, j=1, \ldots, p$.

THEOREM 3.1. Assume that Conditions (C1) and (C2) hold and $\log p=$ $o\left\{\min \left(w_{n}, n w_{n}^{-2}\right)\right\}$. Then (3.13) holds.

Theorem 3.1 shows that, to ensure the accuracy of the normal distribution calibration, the number of simultaneous tests can be as large as $\exp \left\{o\left(n^{1 / 3}\right)\right\}$, when taking $w_{n} \asymp n^{1 / 3}$. We are also interested in estimating FDP in the high dimensional sparse setting, that is, $p$ is large, but the number of $\mu_{j} \neq 0$ is relatively small. The following result indicates that $\operatorname{FDP}_{\mathrm{N}}(z)$ given in (3.9) provides a consistent estimator of the realized FDP in a uniform sense. 
TheOREM 3.2. Assume that Conditions (C1)-(C3) hold. Then, for any sequence of positive numbers $m_{p} \leq 2 p$ satisfying $m_{p} \rightarrow \infty$, we have as $(n, p) \rightarrow \infty$,

$$
\max _{0 \leq z \leq \Phi^{-1}\left(1-m_{p} /(2 p)\right)}\left|\frac{\operatorname{FDP}(z)}{\operatorname{FDP}_{\mathrm{N}}(z)}-1\right| \rightarrow 0 \quad \text { in probability. }
$$

Further, Theorem 3.3 shows that the proposed robust dependence-adjusted inference procedure controls the FDP at a given level $\alpha$ asymptotically with $P$-values estimated from the standard normal distribution.

THEOREM 3.3. Assume that Conditions (C1)-(C4) hold. Then, for any prespecified $0<\alpha<1$,

$$
\left(p_{0} / p\right)^{-1} \operatorname{FDP}\left(\widehat{z}_{\mathrm{N}, 0}\right) \rightarrow \alpha \quad \text { in probability }
$$

as $(n, p) \rightarrow \infty$, where $\widehat{z}_{\mathrm{N}, 0}$ is defined in (3.12).

The constraint on $p$, as a function of $n$, imposed in Theorems 3.2 and 3.3 can be relaxed in a strict factor model with independent idiosyncratic errors.

(C5) $v_{1}, \ldots, v_{p}$ in model (1.6) are independent. As $n, p \rightarrow \infty, p_{0} / p \rightarrow \pi_{0} \in$ $(0,1], \log p=o\left(w_{n}\right)$ and $w_{n}=O\left(n^{1 / 3}\right)$, where $w_{n}$ is as in Condition (C2).

THEOREM 3.4. Assume that Conditions (C1), (C2), (C4) and (C5) hold. Then, for any prespecified $0<\alpha<1,\left(p_{0} / p\right)^{-1} \operatorname{FDP}\left(\widehat{z}_{\mathrm{N}, 0}\right) \rightarrow \alpha$ in probability as $(n, p) \rightarrow \infty$, where $\widehat{z}_{\mathrm{N}, 0}$ is defined in (3.12).

Theorems 3.2-3.4 provide theoretical guarantees on the FDP control for the B$\mathrm{H}$ procedure with dependence-adjusted $P$-values $P_{j}=2 \Phi\left(-\left|T_{j}\right|\right), j=1, \ldots, p$. A similar approach can be defined by using the median-of-means approach, namely, replacing $T_{j}$ 's with $S_{j}$ 's in the definition of $\operatorname{FDP}_{\mathrm{N}}(z)$ in (3.9), which is equivalent to the B-H procedure with $P$-values $Q_{j}=2 \Phi\left(-\left|S_{j}\right|\right), j=1, \ldots, p$. Under similar conditions, the theoretical results on the FDP control remain valid.

THEOREM 3.5. Let $\operatorname{FDP}(z)$ and $\widehat{z}_{\mathrm{N}, 0}$ be defined in (3.8) and (3.12) with $T_{j}$ 's replaced by $S_{j}$ 's, and let $V=V_{n}$ in (3.5) satisfy $V \asymp w_{n}$ for $w_{n}$ as in Condition (C2). Moreover, let $\tau=\tau_{n}$ be as in Condition (C2):

(i) Under Conditions (C1), (C3) and (C4), (3.15) holds for any prespecified $0<\alpha<1$.

(ii) Under Conditions (C1), (C4) and (C5), (3.15) holds for any prespecified $0<\alpha<1$. 


\section{Numerical study.}

4.1. Implementation. To implement the proposed procedure, we solve the convex program (3.2) by using our own implementation in Matlab of the traditional method of scoring, which is an iterative method starting at an initial estimate $\widehat{\boldsymbol{\theta}}^{0} \in \mathbb{R}^{K+1}$. Here, we take $\widehat{\boldsymbol{\theta}}^{0}=\mathbf{0}$; using the current estimate $\widehat{\boldsymbol{\theta}}^{t}$ at iteration $t=0,1,2, \ldots$, we update the estimate by the Newton-Raphson step:

$$
\widehat{\boldsymbol{\theta}}^{t+1}=\widehat{\boldsymbol{\theta}}^{t}+\left\{\frac{1}{n} \sum_{i=1}^{n} \ell_{\tau}^{\prime \prime}\left(z_{i}^{t}\right)\right\}^{-1}\left(\mathbb{G}^{\top} \mathbb{G}\right)^{-1} \mathbb{G}^{\top}\left(\ell_{\tau}^{\prime}\left(z_{1}^{t}\right), \ldots, \ell_{\tau}^{\prime}\left(z_{n}^{t}\right)\right)^{\top},
$$

where $z^{t}=\left(X_{1 j}, \ldots, X_{n j}\right)^{\top}-\mathbb{G} \widehat{\boldsymbol{\theta}}^{t}$ and $\mathbb{G}=\left(\boldsymbol{g}_{1}, \ldots, \boldsymbol{g}_{n}\right)^{\top} \in \mathbb{R}^{n \times(K+1)}$ with $\boldsymbol{g}_{i}=$ $\left(1, \mathbf{f}_{i}^{\top}\right)^{\top}$.

For each $1 \leq j \leq p$, we apply the above algorithm with $\tau=\tau_{j}:=$ $c \widehat{\sigma}_{j} \sqrt{n / \log (n p)}$ to obtain $\left(\widehat{\mu}_{j}, \widehat{\boldsymbol{b}}_{j}^{\mathrm{T}}\right)^{\mathrm{T}}$, where $\widehat{\sigma}_{j}^{2}$ denotes the sample variance of the fitted residuals using OLS and $c>0$ is a control parameter. We take $c=2$ in all the simulations reported below. In practice, we can use a cross-validation procedure to pick $c$ from only a few candidates, say $\{0.5,1,2\}$.

4.2. Simulations via a synthetic factor model. In this section, we perform Monte Carlo simulations to illustrate the performance of the robust test statistic under approximate factor models with general errors. Consider the Fama-French three factor model

$$
X_{i j}=\mu_{j}+\boldsymbol{b}_{j}^{\top} \mathbf{f}_{i}+u_{i j}, \quad i=1, \ldots, n,
$$

where $\mathbf{u}_{i}=\left(u_{i 1}, \ldots, u_{i p}\right)^{\top}$ are i.i.d. copies of $\mathbf{u}=\left(u_{1}, \ldots, u_{p}\right)^{\top}$. We simulate $\left\{\boldsymbol{b}_{j}\right\}_{j=1}^{p}$ and $\left\{\mathbf{f}_{i}\right\}_{i=1}^{n}$ independently from $N_{3}\left(\boldsymbol{\mu}_{B}, \boldsymbol{\Sigma}_{B}\right)$ and $N_{3}\left(\mathbf{0}, \boldsymbol{\Sigma}_{f}\right)$, respectively. To make the model more realistic, parameters are calibrated from the daily returns of S\&P 500's top 100 constituents (chosen by market cap), for the period from July 1, 2008 to June 29, 2012.

To generate dependent errors, we set $\boldsymbol{\Sigma}_{u}=\operatorname{cov}(\mathbf{u})$ to be a block diagonal matrix where each block is four-by-four correlation matrix with equal off-diagonal entries generated from Uniform $[0,0.5]$. The hypothesis testing is carried out under the alternative: $\mu_{j}=\mu$ for $1 \leq j \leq \pi_{1} p$ and $\mu_{j}=0$ otherwise. In the simulations reported here, the ambient dimension $p=2000$, the proportion of true alternatives $\pi_{1}=0.25$ and the sample size $n$ takes values in $\{80,120\}$. For simplicity, we set $\lambda=0.5$ in our procedure and use the Matlab package mafdr to compute the estimate $\widehat{\pi}_{0}(\lambda)$ of $\pi_{0}=1-\pi_{1}$. For each test, the empirical false discovery rate (FDR) is calculated based on 500 replications with FDR level $\alpha$ taking values in $\{5 \%, 10 \%, 20 \%\}$. The errors $\left\{\mathbf{u}_{i}\right\}_{i=1}^{n}$ are generated independently from the following distributions:

- Model 1. $\mathbf{u} \sim N\left(\mathbf{0}, \boldsymbol{\Sigma}_{u}\right)$ : Centered normal random errors with covariance matrix $\boldsymbol{\Sigma}_{u}$ 
- Model 2. $\mathbf{u} \sim(1 / \sqrt{5}) t_{2.5}\left(\mathbf{0}, \boldsymbol{\Sigma}_{u}\right)$ : Symmetric and heavy-tailed errors following a multivariate $t$-distribution with degrees of freedom 2.5 and covariance matrix $\Sigma_{u}$

- Model 3. $\mathbf{u}=0.5 \mathbf{u}_{\mathrm{N}}+0.5\left(\mathbf{u}_{\mathrm{LN}}-\mathbb{E} \mathbf{u}_{\mathrm{LN}}\right)$, where $\mathbf{u}_{\mathrm{N}} \sim N\left(\mathbf{0}, \boldsymbol{\Sigma}_{u}\right)$ and $\mathbf{u}_{\mathrm{LN}} \sim$ $\exp \left\{N\left(\mathbf{0}, \boldsymbol{\Sigma}_{u}\right)\right\}$ is independent of $\mathbf{u}_{\mathrm{N}}$. This model admits asymmetric and heavytailed errors;

- Model 4. $\mathbf{u}=0.25 \mathbf{u}_{t}+0.75\left(\mathbf{u}_{\mathrm{W}}-\mathbb{E} \mathbf{u}_{\mathrm{W}}\right)$, where $\mathbf{u}_{t} \sim t_{4}\left(\mathbf{0}, \boldsymbol{\Sigma}_{u}\right)$ and the $p$ coordinates of $\mathbf{u}_{\mathrm{W}}$ are i.i.d. random variables following the Weibull distribution with shape parameter 0.75 and scale parameter 0.75 .

The proposed Robust Dependence-Adjusted (RD-A) testing procedure is compared with the Ordinary Dependence-Adjusted (OD-A) procedure that uses OLS to estimate unknown parameters in the factor model, and also with the naive procedure where we directly perform multiple marginal $t$-tests ignoring the common factors. We use RD- $\mathrm{A}_{\mathrm{N}}$ and RD- $\mathrm{A}_{\mathrm{B}}$ to denote the RD-A procedure with normal and bootstrap calibration. The number of bootstrap replications is set to be $B=2000$. The signal strength $\mu$ is taken to be $\sqrt{2(\log p) / n}$ for Models 1,2 and 4 , and $\sqrt{3(\log p) / n}$ for Model 3. Define the false negative rate FNR $=\mathbb{E}\{T /(p-R)\}$, where $T$ is the number of falsely accepted null hypotheses and $R$ is the number of discoveries. The true positive rate (TPR) is defined as the average ratio between the number of correct rejections and $p_{1}=\pi_{1} p$. Empirical FDR, FNR and TPR for the RD-A $A_{B}, R D-A_{N}$, OD-A and naive procedures under different scenarios are presented in Tables 1 and 2. To save space, we leave the numerical comparison between the RD- $\mathrm{A}_{\mathrm{N}}$ and OD-A procedures under some additional models in the supplementary material [Zhou et al. (2018)], along with comparisons across a range of sample sizes and signal strengths.

For weakly dependent errors following the normal distribution and $t$ distribution, Table 1 shows that the RD-A procedure consistently outperforms the OD-A method, in the sense that the RD-A method provides a much better control of the FDR at the expense of slight compromises of the FNR and TPR. Should the FDR being controlled at the same level, the robust method will be more powerful. In Table 2, when the errors are both asymmetric and heavy-tailed, the RD-A procedure has the biggest advantage in that it significantly outperforms the OD-A on controlling the FDR at all levels while maintaining low FNR and high TPR. Together, these results show that the RD-A procedure is indeed robust to outliers and does not lose efficiency when the errors are symmetric and light-tailed. In terms of controlling FDR, Models 3 and 4 present more challenges than Models 1 and 2 due to being both heavy-tailed and asymmetric. In Table 2, we see that although both the RD-A and OD-A methods achieve near-perfect power, the empirical FDR is higher than the desired level across all settings and much more higher for OD-A. Hence we compare the FDR of the RD-A and OD-A methods for various sample sizes in Figure 2. We see that the empirical FDR decreases with increase in sample size, while consistently outperforming the OD-A procedure. The difference 
TABLE 1

Empirical FDR, FNR and TPR based on a factor model with dependent errors following a normal distribution (Model 1) and a $t$-distribution (Model 2)

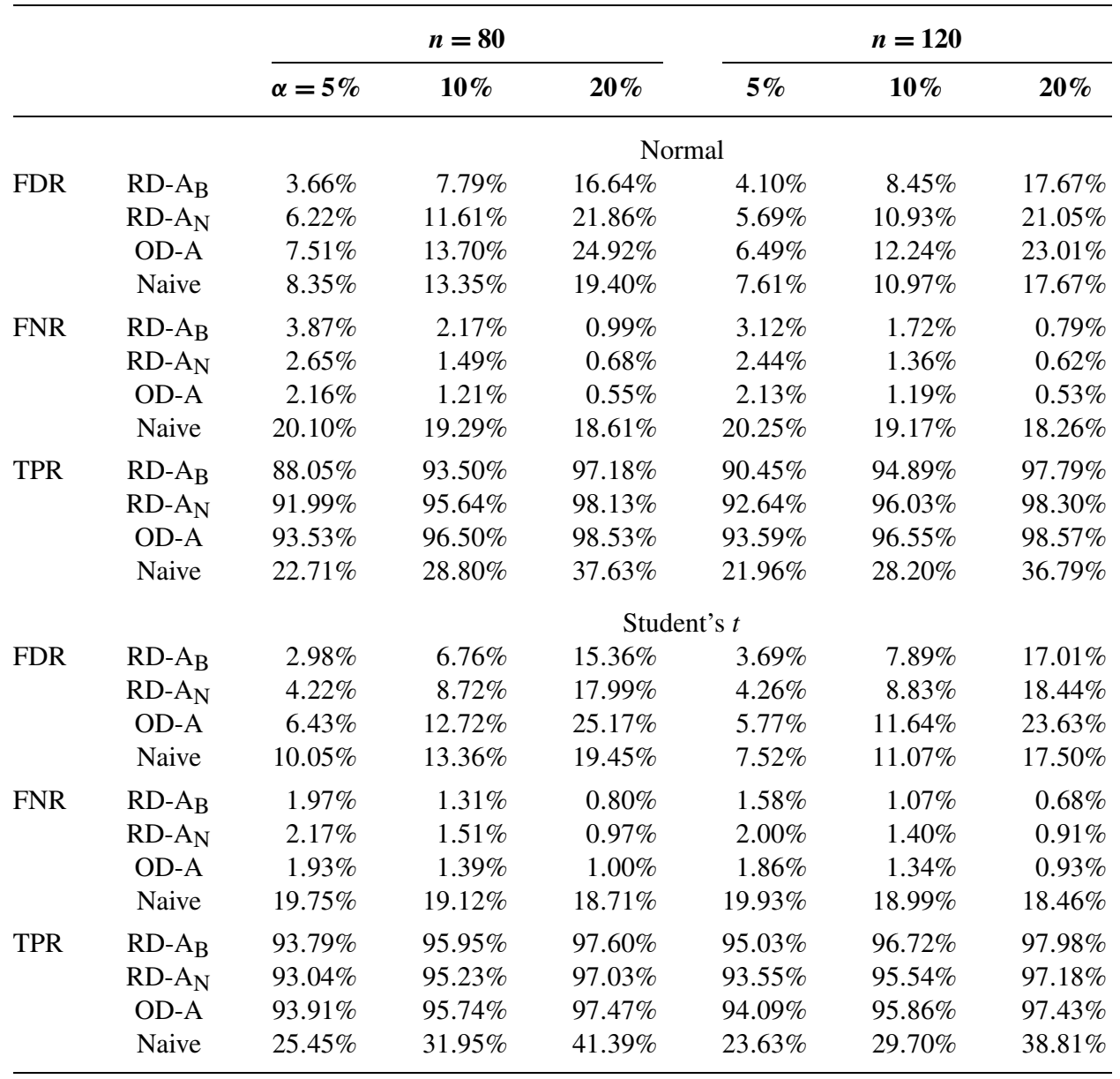

between the two methods is greater for lower sample sizes, reinforcing the usefulness of our method for high dimensional heavy-tailed data with moderate sample sizes.

The naive procedure suffers from a significant loss in FNR and TPR. The reasons are twofold: (a) the naive procedure ignores the actual dependency structure among the variables; (b) the signal-to-noise ratio of $H_{0 j}: \mu_{j}=0$ for the naive procedure is $\sigma_{j j}^{-1 / 2}\left|\mu_{j}\right|$, which can be much smaller than $\sigma_{v, j j}^{-1 / 2}\left|\mu_{j}\right|$ for the dependence-adjusted procedure.

4.3. Stock market data. In this section, we apply our proposed robust dependence-adjusted multiple testing procedure to monthly stock market data. 
TABLE 2

Empirical FDR, FNR and TPR based on a factor model with dependent errors following a mixture normal/lognormal distribution (Model 3) and a mixture Student's t/Weibull distribution (Model 4)

\begin{tabular}{|c|c|c|c|c|c|c|c|}
\hline & & & $n=80$ & & & $n=120$ & \\
\hline & & $\alpha=5 \%$ & $10 \%$ & $20 \%$ & $5 \%$ & $10 \%$ & $20 \%$ \\
\hline & & & & xture Nor & Lognorm & & \\
\hline FDR & $\mathrm{RD}-\mathrm{A}_{\mathrm{B}}$ & $8.40 \%$ & $13.33 \%$ & $22.04 \%$ & $8.02 \%$ & $12.98 \%$ & $21.99 \%$ \\
\hline & $\mathrm{RD}-\mathrm{A}_{\mathrm{N}}$ & $10.29 \%$ & $16.02 \%$ & $25.87 \%$ & $9.16 \%$ & $14.65 \%$ & $24.61 \%$ \\
\hline & OD-A & $12.18 \%$ & $18.46 \%$ & $29.24 \%$ & $10.28 \%$ & $16.17 \%$ & $26.79 \%$ \\
\hline & Naive & $7.79 \%$ & $12.18 \%$ & $18.49 \%$ & $7.99 \%$ & $11.77 \%$ & $17.93 \%$ \\
\hline FNR & RD-A $A_{B}$ & $0.32 \%$ & $0.09 \%$ & $0.02 \%$ & $0.29 \%$ & $0.11 \%$ & $0.03 \%$ \\
\hline & $R D-A_{N}$ & $0.57 \%$ & $0.25 \%$ & $0.07 \%$ & $0.56 \%$ & $0.23 \%$ & $0.06 \%$ \\
\hline & OD-A & $0.26 \%$ & $0.08 \%$ & $0.02 \%$ & $0.29 \%$ & $0.10 \%$ & $0.03 \%$ \\
\hline & Naive & $18.66 \%$ & $16.99 \%$ & $15.26 \%$ & $18.51 \%$ & $16.67 \%$ & $14.78 \%$ \\
\hline TPR & RD-A $A_{B}$ & $99.07 \%$ & $99.73 \%$ & $99.95 \%$ & $99.15 \%$ & $99.70 \%$ & $99.92 \%$ \\
\hline & $R D-A_{N}$ & $98.36 \%$ & $99.31 \%$ & $99.81 \%$ & $98.38 \%$ & $99.36 \%$ & $99.82 \%$ \\
\hline & OD-A & $99.26 \%$ & $99.76 \%$ & $99.96 \%$ & $99.16 \%$ & $99.72 \%$ & $99.93 \%$ \\
\hline & Naive & $28.39 \%$ & $37.37 \%$ & $50.14 \%$ & $29.72 \%$ & $39.34 \%$ & $51.95 \%$ \\
\hline & & & & xture Stud & s $t /$ Weibu & & \\
\hline FDR & $\mathrm{RD}-\mathrm{A}_{\mathrm{B}}$ & $7.51 \%$ & $12.05 \%$ & $20.43 \%$ & $7.03 \%$ & $11.66 \%$ & $20.49 \%$ \\
\hline & RD- $A_{N}$ & $8.91 \%$ & $14.34 \%$ & $24.11 \%$ & $7.85 \%$ & $13.17 \%$ & $23.11 \%$ \\
\hline & OD-A & $11.00 \%$ & $17.11 \%$ & $27.67 \%$ & $9.09 \%$ & $14.80 \%$ & $25.37 \%$ \\
\hline & Naive & $9.33 \%$ & $13.48 \%$ & $20.30 \%$ & $8.14 \%$ & $12.52 \%$ & $18.85 \%$ \\
\hline & RD-A $A_{B}$ & $0.62 \%$ & $0.23 \%$ & $0.06 \%$ & $0.62 \%$ & $0.25 \%$ & $0.08 \%$ \\
\hline FNR & $R D-A_{N}$ & $0.62 \%$ & $0.24 \%$ & $0.06 \%$ & $0.60 \%$ & $0.24 \%$ & $0.07 \%$ \\
\hline & OD-A & $0.30 \%$ & $0.11 \%$ & $0.03 \%$ & $0.42 \%$ & $0.16 \%$ & $0.05 \%$ \\
\hline & Naive & $19.91 \%$ & $18.78 \%$ & $18.08 \%$ & $20.71 \%$ & $20.02 \%$ & $19.36 \%$ \\
\hline TPR & $\mathrm{RD}-\mathrm{A}_{\mathrm{B}}$ & $98.16 \%$ & $99.34 \%$ & $99.83 \%$ & $98.16 \%$ & $99.29 \%$ & $99.78 \%$ \\
\hline & $\mathrm{RD}-\mathrm{A}_{\mathrm{N}}$ & $98.18 \%$ & $99.31 \%$ & $99.82 \%$ & $98.22 \%$ & $99.30 \%$ & $99.80 \%$ \\
\hline & OD-A & $99.14 \%$ & $99.69 \%$ & $99.92 \%$ & $98.78 \%$ & $99.54 \%$ & $99.86 \%$ \\
\hline & Naive & $23.41 \%$ & $30.24 \%$ & $39.65 \%$ & $20.49 \%$ & $25.90 \%$ & $34.07 \%$ \\
\hline
\end{tabular}

Consider Carhart's four-factor model [Carhart (1997)] on the S\&P 500 index, where the excess return of a stock has the following representation:

$$
\begin{aligned}
r_{j t}= & \mu_{j}+\beta_{j, \mathrm{MKT}}\left(\mathrm{MKT}_{t}-r_{f t}\right)+\beta_{j, \mathrm{SMB}} \mathrm{SMB}_{t} \\
& +\beta_{j, \mathrm{HML}} \mathrm{HML}_{t}+\beta_{j, \mathrm{UMD}} \mathrm{UMD}_{t}+u_{j t},
\end{aligned}
$$

for $j=1, \ldots, p$ and $t=1, \ldots, T$. Here, $r_{j t}$ is the excess returns of stock $j$ at month $t, r_{f t}$ is the risk-free interest rate at month $t$, and MKT, HML, SMB and UMD represent the market, value, size and momentum factors, respectively. We are interested in the value $\mu_{j}$, which represents the alpha of stock $j$. A stock can be said to have excess returns if its alpha is positive, or in other words, the stock 

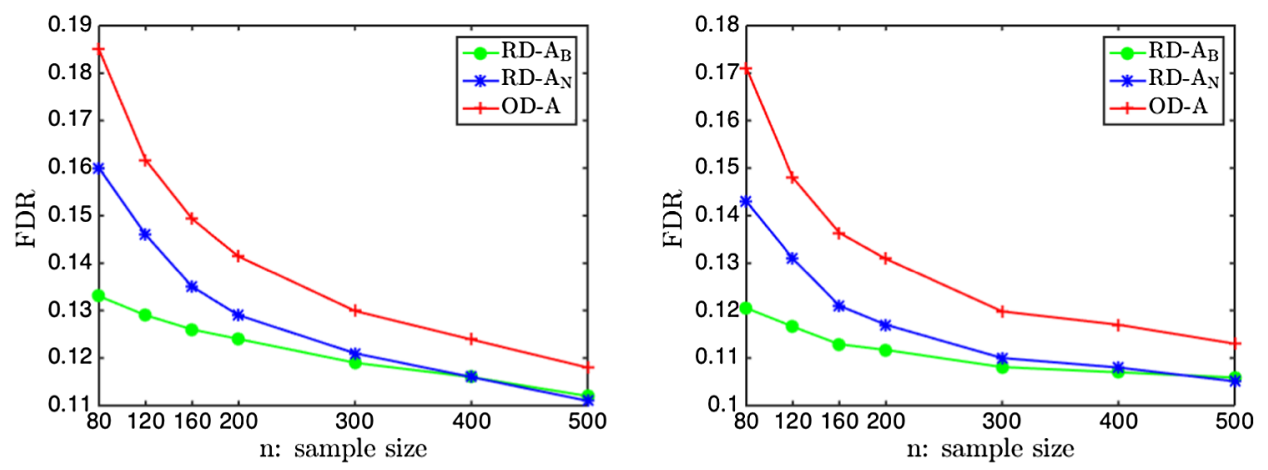

FIG. 2. Empirical FDR of the testing problem at the $10 \%$ significance level, when the data follows a mixture normal/lognormal distribution (Model 3) on the left panel and a mixture Student's $t /$ Weibull distribution (Model 4) on the right panel, and the sample size varies.

exhibits returns higher than those that can be accounted for by the four factors. If the alpha is negative, the stock is consistently underperforming, given the level of risk it undertakes. Detecting nonzero alpha is important since it is directly related to the efficient equity market hypothesis. When the market is inefficient, we can conduct multiple hypothesis testing to identify those stocks in the market that have statistically significant alphas. When the returns of mutual fund data are used, the test is related to test whether the fund manager has skills or not [Barras, Scaillet and Wermers (2010)]. All the data in this section was obtained from Kenneth French's website and the COMPUSTAT and CRSP databases.

We obtain monthly data for 393 S\&P 500 constituents over the time period from January 2005 to December 2013, after removing those stocks that have missing values or have discontinuous inclusion in the index. The stock returns exhibit severely heavy-tails, as illustrated by the histogram of the excess kurtosis of the data in Figure 3. Among the 393 series, 112 have distributions whose tails are fatter than the $t$-distribution with 5 degrees of freedom.

The regression in (4.2) is carried out over rolling windows: for each month, we evaluate the model using data from the preceding three years. For each rolling window, we simultaneously test the hypotheses $H_{0 j}: \mu_{j}=0$ versus $H_{1 j}: \mu_{j} \neq 0$ for $j=1, \ldots, p$, using the proposed robust dependence-adjusted procedure. We see that out of a portfolio of size 393, only a few stocks exhibit statistically significant nonzero alphas at the FDR threshold of 5\%,10\% and 20\%. Table 3 summarizes the results for the number of selected stocks and estimated alphas with the FDR controlled at 5\%. For the robust dependence-adjusted procedure, we see that no stock is selected on average, with a maximum of 2 stocks selected over the entire time period. In particular, our method does not select any stocks from the third quarter of 2008 to the third quarter of 2010, coinciding with the financial crisis during which the market volatility is much higher. Moreover, the estimated alphas for the selected stocks are much higher than those not selected by the robust multiple 


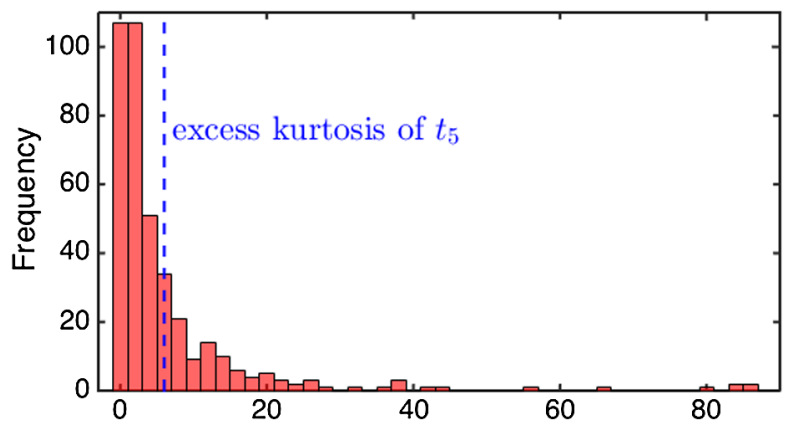

FIG. 3. Histogram of excess kurtosises of monthly returns of 393 S\&P 500 constituents from January 2005 to December 2013. The excess kurtosis of the $t_{5}$ distribution is shown for reference.

testing procedure. This is represented as $\left|\widehat{\mu}_{j}\right|$ in Table 3. The naive method, which directly performs multiple $t$-tests ignoring the common factors, appears to be unstable with the number of stocks selected being extremely variable. Additionally, a tremendously large number of stocks are selected in a few time periods, pointing toward false discoveries. In summary, the robust dependence-adjusted multiple testing procedure is particularly suited for the problem of finding a few stocks with nonzero alphas, which is explained by the focus on a balanced panel of highly traded stocks with large capitalizations, namely, the constituents of the S\&P 500.

4.4. Gene expression data. In this section, we apply the proposed procedure to the analysis of a neuroblastoma data set reported in Oberthuer et al. (2006) to identify differentially expressed genes between the group of patients who had 3year event-free survival after the diagnosis of neuroblastoma and the group of pa-

\section{TABLE 3}

Summary of the three testing procedures based on 393 stocks in S\&P 500 between January 2005 and December 2013. A rolling window of 3 years is used for estimation and selecting stocks with significant nonzero alpha. The stocks are selected at FDR level 5\%

\begin{tabular}{lcccccc}
\hline Variable & Method & Mean & Std. Dev. & Median & Min & Max \\
\hline Number of selected stocks & RD-A & 0.18 & 0.42 & 0 & 0 & 2 \\
& OD-A & 0.94 & 1.59 & 0 & 0 & 7 \\
& Naive & 4.39 & 22.46 & 0 & 0 & 178 \\
$\left|\widehat{\mu}_{j}\right|$ for selected stocks & RD-A & $4.19 \%$ & $1.41 \%$ & $4.31 \%$ & $2.43 \%$ & $6.58 \%$ \\
& OD-A & $3.37 \%$ & $1.00 \%$ & $3.24 \%$ & $1.70 \%$ & $6.34 \%$ \\
& Naive & $3.76 \%$ & $1.20 \%$ & $3.47 \%$ & $2.77 \%$ & $8.01 \%$ \\
$\left|\widehat{\mu}_{j}\right|$ for nonselected stocks & RD-A & $1.01 \%$ & $0.09 \%$ & $1.00 \%$ & $0.90 \%$ & $1.25 \%$ \\
& OD-A & $1.09 \%$ & $0.11 \%$ & $1.08 \%$ & $0.91 \%$ & $1.32 \%$ \\
& Naive & $1.75 \%$ & $0.35 \%$ & $1.63 \%$ & $1.35 \%$ & $2.45 \%$ \\
\hline
\end{tabular}


tients who did not. This data set consists of 251 patients of the German Neuroblastoma Trials NB90-NB2004, diagnosed between 1989 and 2004. The complete data set, obtained via the MicroArray Quality Control phase-II (MAQC-II) project [Shi et al. (MAQC Consortium) (2010)], includes gene expression over 10,707 probe sites. There are 246 subjects with 3-year event-free survival information available (56 positive and 190 negative). See Oberthuer et al. (2006) for more details about the data sets.

In the first stage, we use standard principal component analysis on the two samples to obtain the factors, based on which we construct dependence-adjusted $P$ values to conduct multiple testing in the second step. Note that the test statistic given in (3.7) can be directly generalized to the two-sample case: Given two groups of $p$-dimensional ( $p=10,707)$ observations with sizes $n_{1}=56$ and $n_{2}=190$, we compute robust mean and variance estimators $\left(\widehat{\mu}_{1 j}, \widehat{\mu}_{2 j}\right)$ and $\left(\widehat{\sigma}_{1 v, j j}, \widehat{\sigma}_{2 v, j j}\right)$ for $j=1, \ldots, p$. Define two-sample test statistics $T_{j}=\left(\widehat{\mu}_{1 j}-\widehat{\mu}_{2 j}\right) /\left(\widehat{\sigma}_{1 v, j j} / n_{1}+\right.$ $\left.\widehat{\sigma}_{2 v, j j} / n_{2}\right)^{1 / 2}$ so that the corresponding $P$-values are $\left\{2 \Phi\left(-\left|T_{j}\right|\right)\right\}_{j=1}^{p}$.

The number of factors is estimated by the eigenvalue ratio estimator proposed in Ahn and Horenstein (2013), which was also used in the context of factoradjusted multiple testing in Fan and Han (2017). The estimator is defined as $\widehat{K}=\operatorname{argmax}_{1<k<k_{\max }}\left(\widehat{\lambda}_{k} / \widehat{\lambda}_{k+1}\right)$, where $\widehat{\lambda}_{j}$ is the $j$ th eigenvalue of the sample covariance matrix and $k_{\max }$ is the maximum possible number of factors. Following this procedure, we use $K=2$ to model the latent structure in the data.

Next, we conduct multiple testing using the proposed robust dependenceadjusted procedure and the naive procedure based on two-sample $t$-tests. At FDR level $1 \%$, we detect 3779 genes and the naive procedure detects 3236 genes; while at FDR level 5\%, we discover 5223 genes and the naive procedure discovers 4685 genes. In general, taking the latent structure into account causes a visible increase in the number of genes that are declared statistically significant regardless of the prechosen FDR level, reflecting the improved power of our method. This phenomenon is in accord with that in Desai and Storey (2012). These results may serve as an exploratory step for more refined analyses regarding those significant genes.

5. Summary and discussion. This paper consists of two main parts with each one being of independent interest. In the first part, we study the conventional robust $M$-estimation [Huber (1973)] from a new perspective by allowing the robustification parameter $\tau$ to diverge with the sample size to balance the bias and robustness of the estimator. Our main theoretical contribution (Theorem 2.1) is a nonasymptotic Bahadur representation of the proposed robust estimator along with a subGaussian-type deviation bound if the error variable has a finite second moment. As by-products, we prove the Berry-Esseen inequality and a Cramér-type moderate deviation theorem for the estimator. These probabilistic results are particularly useful in investigating robustness and accuracy of the $P$-values in multiple testing, 
among other high dimensional statistical inference problems [Fan, Hall and Yao (2007), Delaigle, Hall and Jin (2011), Chang, Shao and Zhou (2016)].

In the second part, we focus on large-scale multiple testing for dependent and heavy-tailed data. To characterize the dependence, we employ a multi-factor model similar to that used in Desai and Storey (2012), Fan, Han and Gu (2012) and Fan and Han (2017) but with an observable factor. To achieve robustness, we propose a Huber loss based approach to construct test statistics for testing the individual hypotheses. Under mild conditions, our procedure asymptotically controls the overall false discovery proportion at the nominal level. Thorough numerical results on both simulated and real world datasets are also provided to back up our theory. It is shown that the newly proposed robust dependence-adjusted method performs well numerically in terms of both the size and power. It significantly outperforms the multiple $t$-tests under strong dependence, and is applicable even when the true error distribution deviates wildly from the normal distribution. A more interesting and challenging problem is when the dependence structure is characterized by latent factors. In this case, robust estimators of the unobservable factors along with the loadings are required. Large-scale simultaneous inference for latent factor models with heavy-tailed errors is our ongoing work. We leave the details of the results elsewhere in the future.

Acknowledgments. We would like to thank the Editor, Dr. Tailen Hsing, the Associate Editor and three reviewers for their constructive comments which helped us improve the paper, and Dr. Qiang Sun for helpful discussions.

\section{SUPPLEMENTARY MATERIAL}

Supplement to "A new perspective on robust $M$-estimation: Finite sample theory and applications to dependence-adjusted multiple testing" (DOI: 10.1214/17-AOS1606SUPP; .pdf). This supplemental material contains the proofs for the theoretical results in the main text and additional simulation results.

\section{REFERENCES}

Ahn, S. C. and Horenstein, A. R. (2013). Eigenvalue ratio test for the number of factors. Econometrica 81 1203-1227. MR3064065

BARBE, P. and BERTAIL, P. (1995). The Weighted Bootstrap. Lecture Notes in Statistics 98. Springer, New York. MR2195545

Barras, L., Scaillet, O. and Wermers, R. (2010). False discoveries in mutual fund performance: Measuring luck in estimated alphas. J. Finance 65 179-216.

Benjamini, Y. and Hochberg, Y. (1995). Controlling the false discovery rate: A practical and powerful approach to multiple testing. J. R. Stat. Soc. Ser. B. Stat. Methodol. 57 289-300. MR1325392

Benjamini, Y. and YeKUTIELI, D. (2001). The control of the false discovery rate in multiple testing under dependency. Ann. Statist. 29 1165-1188. MR1869245

CARHART, M. M. (1997). On persistence in mutual fund performance. J. Finance 52 57-82. 
CAtoni, O. (2012). Challenging the empirical mean and empirical variance: A deviation study. Ann. Inst. Henri Poincaré Probab. Stat. 48 1148-1185. MR3052407

Chang, J., Shao, Q.-M. and Zhou, W.-X. (2016). Cramér-type moderate deviations for Studentized two-sample $U$-statistics with applications. Ann. Statist. 44 1931-1956. MR3546439

Clarke, S. and Hall, P. (2009). Robustness of multiple testing procedures against dependence. Ann. Statist. 37 332-358. MR2488354

CONT, R. (2001). Empirical properties of asset returns: Stylized facts and statistical issues. Quant. Finance $1223-236$.

Delaigle, A., Hall, P. and Jin, J. (2011). Robustness and accuracy of methods for high dimensional data analysis based on Student's $t$-statistic. J. R. Stat. Soc. Ser. B. Stat. Methodol. 73 283-301. MR2815777

Desai, K. H. and Storey, J. D. (2012). Cross-dimensional inference of dependent highdimensional data. J. Amer. Statist. Assoc. 107 135-151. MR2949347

EFron, B. (2004). Large-scale simultaneous hypothesis testing: The choice of a null hypothesis. J. Amer. Statist. Assoc. 99 96-104. MR2054289

EFron, B. (2007). Correlation and large-scale simultaneous significance testing. J. Amer. Statist. Assoc. 102 93-103. MR2293302

Efron, B., Tibshirani, R., Storey, J. D. and Tusher, V. (2001). Empirical Bayes analysis of a microarray experiment. J. Amer. Statist. Assoc. 96 1151-1160. MR1946571

FAMA, E. F. (1963). Mandelbrot and the stable paretian hypothesis. J. Bus. 36 420-429.

FAN, J., HALL, P. and YAO, Q. (2007). To how many simultaneous hypothesis tests can normal, Student's $t$ or bootstrap calibration be applied? J. Amer. Statist. Assoc. 102 1282-1288. MR2372536

FAN, J. and HAN, X. (2017). Estimation of the false discovery proportion with unknown dependence. J. R. Stat. Soc. Ser. B. Stat. Methodol. 79 1143-1164.

FAN, J., HAN, X. and GU, W. (2012). Estimating false discovery proportion under arbitrary covariance dependence. J. Amer. Statist. Assoc. 107 1019-1035. MR3010887

FAN, J., LI, Q. and WANG, Y. (2017). Estimation of high dimensional mean regression in the absence of symmetry and light tail assumptions. J. R. Stat. Soc. Ser. B. Stat. Methodol. 79 247-265. MR3597972

Finkenstadt, B. and RootzeÉn, H. (2003). Extreme Values in Finance, Telecommunications and the Environment. Chapman \& Hall, New York.

Friguet, C., KLOAREg, M. and CAUSEUR, D. (2009). A factor model approach to multiple testing under dependence. J. Amer. Statist. Assoc. 104 1406-1415. MR2750571

Genovese, C. and WASSERMAN, L. (2004). A stochastic process approach to false discovery control. Ann. Statist. 32 1035-1061. MR2065197

HE, X. and SHAO, Q.-M. (1996). A general Bahadur representation of $M$-estimators and its application to linear regression with nonstochastic designs. Ann. Statist. 24 2608-2630. MR1425971

HE, X. and SHAO, Q.-M. (2000). On parameters of increasing dimensions. J. Multivariate Anal. 73 120-135. MR1766124

Huber, P. J. (1964). Robust estimation of a location parameter. Ann. Math. Stat. 35 73-101. MR0161415

Huber, P. J. (1973). Robust regression: Asymptotics, conjectures and Monte Carlo. Ann. Statist. 1 799-821. MR0356373

Joly, E. and Lugosi, G. (2016). Robust estimation of $U$-statistics. Stochastic Process. Appl. 126 3760-3773. MR3565476

KosoroK, M. R. and MA, S. (2007). Marginal asymptotics for the "large $p$, small $n$ " paradigm: With applications to microarray data. Ann. Statist. 35 1456-1486. MR2351093

LANGAas, M., Lindqvist, B. H. and FerkingStad, E. (2005). Estimating the proportion of true null hypotheses, with application to DNA microarray data. J. R. Stat. Soc. Ser. B. Stat. Methodol. 67 555-572. MR2168204 
Leek, J. T. and Storey, J. D. (2008). A general framework for multiple testing dependence. Proc. Natl. Acad. Sci. USA 105 18718-18723.

Lehmann, E. L. and Romano, J. P. (2005). Generalizations of the familywise error rate. Ann. Statist. 33 1138-1154. MR2195631

LINNIK, JU. V. (1961). On the probability of large deviations for the sums of independent variables. In Proc. 4th Berkeley Sympos. Math. Statist. and Prob., Vol. II 289-306. Univ. California Press, Berkeley, CA. MR0137142

LiU, W. and SHAO, Q.-M. (2010). Cramér-type moderate deviation for the maximum of the periodogram with application to simultaneous tests in gene expression time series. Ann. Statist. 38 1913-1935. MR2662363

LiU, W. and SHAO, Q.-M. (2014). Phase transition and regularized bootstrap in large-scale $t$-tests with false discovery rate control. Ann. Statist. 42 2003-2025. MR3262475

MAMMEN, E. (1989). Asymptotics with increasing dimension for robust regression with applications to the bootstrap. Ann. Statist. 17 382-400. MR0981457

Mandelbrot, B. (1963). The variation of certain speculative prices. J. Bus. 36 394-419.

Meinshausen, N. and RiCE, J. (2006). Estimating the proportion of false null hypotheses among a large number of independently tested hypotheses. Ann. Statist. 34 373-393. MR2275246

Nemirovsky, A. S. and Yudin, D. B. (1983). Problem Complexity and Method Efficiency in Optimization. Wiley, New York. MR0702836

Oberthuer, A., Berthold, F., Warnat, P., Hero, B., Kahlert, Y., Spitz, R., Ernestus, K., König, R., HaAs, S., Eils, R., Schwab, M., Brors, B., Westermann, F. and FISCHER, M. (2006). Customized oligonucleotide microarray gene expression based classification of neuroblastoma patients outperforms current clinical risk stratification. J. Clin. Oncol. 24 5070-5078.

PORTNOY, S. (1985). Asymptotic behavior of $M$ estimators of $p$ regression parameters when $p^{2} / n$ is large. II. Normal approximation. Ann. Statist. 13 1403-1417. MR0811499

SCHWARTZMAN, A. and LIN, X. (2011). The effect of correlation in false discovery rate estimation. Biometrika 98 199-214. MR2804220

SHI, L., et al. (MAQC CONSORTIUM) (2010). The MicroArray Quality Control (MAQC)-II study of common practices for the development and validation of microarray-based predictive models. Nat. Biotechnol. 28 827-841.

SpOKOINY, V. and ZHILOVA, M. (2015). Bootstrap confidence sets under model misspecification. Ann. Statist. 43 2653-2675. MR3405607

Storey, J. D. (2002). A direct approach to false discovery rates. J. R. Stat. Soc. Ser. B. Stat. Methodol. 64 479-498. MR1924302

Storey, J. D., TAYlor, J. E. and Siegmund, D. (2004). Strong control, conservative point estimation and simultaneous conservative consistency of false discovery rates: A unified approach. J. R. Stat. Soc. Ser. B. Stat. Methodol. 66 187-205. MR2035766

Sun, W. and CAI, T. T. (2009). Large-scale multiple testing under dependence. J. R. Stat. Soc. Ser. B. Stat. Methodol. 71 393-424. MR2649603

VERSHYNIN, R. (2012). Introduction to the non-asymptotic analysis of random matrices. In Compressed Sensing 210-268. Cambridge Univ. Press, Cambridge. MR2963170

Yohai, V. J. and MaronnA, R. A. (1979). Asymptotic behavior of $M$-estimators for the linear model. Ann. Statist. 7 258-268. MR0520237

ZhILOVA, M. (2016). Non-classical Berry-Esseen inequality and accuracy of the weighted bootstrap. Available at arXiv:1611.02686.

Zhou, W.-X., Bose, K., FAn, J. and LiU, H. (2018). Supplement to "A new perspective on robust $M$-estimation: Finite sample theory and applications to dependence-adjusted multiple testing." DOI:10.1214/17-AOS1606SUPP. 
W.-X. ZHOU

DEPARTMENT OF MATHEMATICS

University of CALIFornia, SAN Diego

LA JOLLA, CALIFORNIA 92093

USA

E-MAIL:wez243@ucsd.edu
K. Bose

H. LIU

DEPARTMENT OF OPERATIONS RESEARCH AND FinANCIAL ENGINEERING

PRINCETON UNIVERSITY

Princeton, NeW JeRSEY 08544

USA

E-MAIL: bose@princeton.edu hanliu@princeton.edu

J. FAN

SCHOOL OF DATA SCIENCE

FUDAN UNIVERSITY

SHANGHAI 200433

CHINA

AND

DEPARTMENT OF OPERATIONS RESEARCH AND FINANCIAL ENGINEERING

PRINCETON UNIVERSITY

PRINCETON, NEW JERSEY 08544

USA

E-MAIL: jqfan@princeton.edu 